This item was submitted to Loughborough's Research Repository by the author.

Items in Figshare are protected by copyright, with all rights reserved, unless otherwise indicated.

\title{
Monodromy in the resonant swing spring
}

PLEASE CITE THE PUBLISHED VERSION

LICENCE

CC BY-NC-ND 4.0

\section{REPOSITORY RECORD}

Dullin, Holger R., Andrea Giacobbe, and Richard Cushman. 2019. "Monodromy in the Resonant Swing Spring". figshare. https://hdl.handle.net/2134/298. 


\title{
Monodromy in the resonant swing spring
}

\author{
Holger Dullin ${ }^{1}, \quad$ Andrea Giacobbe ${ }^{2}$, and Richard Cushman ${ }^{2}$
}

\begin{abstract}
This paper shows that an integrable approximation of the spring pendulum, when tuned to be in $1: 1: 2$ resonance, has monodromy. The stepwise precession angle of the swing plane of the resonant spring pendulum is shown to be a rotation number of the integrable approximation. Due to the monodromy, this rotation number is not a globally defined function of the integrals. In fact at lowest order it is given by $\arg (a+i b)$ where $a$ and $b$ are functions of the integrals. The resonant swing spring is therefore a system where monodromy has easily observed physical consequences.
\end{abstract}

\section{Introduction}

The spring pendulum or swing spring is one of the simplest possible mechanical systems. It is a spring with one end fixed and at the other end is attached a mass that is acted on by a constant vertical gravitation field. The name swing spring comes from the fact that for appropriate initial conditions the mass can either swing like a pendulum or bounce up and down like a spring. However, if the frequencies of the swinging and springing motion in linear approximation near the equilibrium are in resonance, these two types of motions are intricately intertwined. In particular, the following motion is easily observed. Starting with the weakly unstable vertical springing motion, the system evolves into a planar swinging motion. This swinging motion is also transient and the system returns to its original springing motion. This cycle then repeats. Lynch [6] observed that the orientation of the swing plane typically changes from one swinging phase to the next. Moreover, the angle between the swing planes of any two successive swinging phases is constant. However, the angle between the swing planes depends on initial conditions. He called this phenomenon the stepwise precession of the swing plane of the swing spring. It is this phenomenon that we are going to explain both qualitatively and quantitatively.

\footnotetext{
${ }^{1}$ Mathematical Sciences, Loughborough University, Loughborough, LE11 3TU U.K.

${ }^{2}$ Mathematics Institute, University of Utrecht, 3508TA Utrecht, The Netherlands
} 
The swing spring has a long history that is well described in [7]. The earliest comprehensive work on the planar spring pendulum is [11]. This paper gives a classical treatment of the $1: 2$ resonance using action angle variables. It is written in the spirit of the old quantum mechanics and was actually motivated by the Fermi 1 : 2 resonance in $\mathrm{CO}_{2}$. The advent of modern quantum mechanics seems to have made this type of analysis old-fashioned if not archaic. As Lynch [7] points out most of the previous work is only concerned with the planar spring pendulum, so that the stepwise precession of the swing plane cannot be found. Some progress on the three dimensional system was made in [6]. After that Holm and Lynch [5] found that the system can be approximated by the 3-waves system and derived a differential equation for the angle of the swing plane. This was done using "pattern evocation in shape space" [8]. We show that equation found by Holm and Lynch is exact and is nothing but the equation for the evolution of one the angles of the action-angle coordinates of an integrable approximation to the resonant swing spring. We then trace the origin of the stepwise precession to the existence of Hamiltonian monodromy in this integrable approximation. Hamiltonian monodromy is an obstruction to the existence of global action variables, which was first described in [4] (see also [2]). It generically appears around an equilibrium point of an integrable two degree of freedom Hamiltonian system whose linearization has a complex quartuple $\pm \alpha \pm i \beta$ of eigenvalues [12]. Such an equilibrium point is of focus-focus type. The integrable approximation of the resonant swing spring has three degrees of freedom. But after reduction of a symmetry, one obtains a two degree of freedom system with a focus-focus point as a relative equilibrium. Physically this corresponds to the pure springing motion of the system. For the purpose of the present paper the most important consequence of monodromy is that the rotation number of invariant tori, that is, the ratio of their frequencies, near the singularity is not a single valued function. Our main result is that the stepwise precession of the swing plane is given by such a rotation number, which explicitly has the form

$$
\Delta \vartheta=\arg (a+i b)+\mathrm{O}\left(\sqrt{a^{2}+b^{2}}\right) .
$$

Here $a$ and $b$ are simple function of the integrals of the system, and $a, b \rightarrow 0$ near the equilibrium of the swing spring. The amazing feature of (1) is that it is not differentiable at the origin. This means that no matter how small the initial perturbation from the equilibrium is, one can always obtain all possible values for $\Delta \vartheta$. In general, such multivaluednes has been described 
for integrable foliations near focus-focus singularitites by $\mathrm{Vu} \mathrm{Ngoc}$ [10]. In some sense our result is a special case of his. However, he did not study the influence of the Hamiltonian, but only the foliation.

The paper is organised as follows. In section 2 we briefly recall the physics of the swing spring. Then we derive an integrable approximation which is valid near the resonant equilibrium point. This integrable system is then reduced to a one degree of freedom system in section 4 . We then describe the geometry of the image of its energy momentum map and show that there is monodromy. The dynamics of the swing angle $\vartheta$ is described in section 6 and finally we obtain equation (1) for the rotation number $\Delta \vartheta$, by approximating an elliptic integral.

\section{The physics of the swing spring}

The spring pendulum is a point particle $r=(x, y, z)$ in $\mathbb{R}^{3}$ of mass $m$ attached to a spring which moves in a constant vertical gravitation field. Its potential energy is

$$
\widetilde{V}(r)=m g z+\frac{1}{2} k\left(\ell_{0}-\|r\|\right)^{2},
$$

where $\|r\|=\sqrt{x^{2}+y^{2}+z^{2}}$. The unstretched length of the spring with spring constant $k$ is $\ell_{0}$ and $g$ is the constant of gravity. The motion of the swing spring is governed by Newton's equations

$$
m \ddot{r}=-\operatorname{grad} \tilde{V}(r) .
$$

The system is in equilibrium when the forces of gravity and the spring balance, that is, when

$$
\operatorname{grad} \widetilde{V}(r)=0
$$

Thus $x=y=0$ and $z=-\ell$. The equilibrium length $\ell$ of the spring is determined by

$$
k\left(\ell-\ell_{0}\right)=m g
$$

Measuring length in units of equilibrium length $\ell$, mass in units of $m$ and time in units of $\sqrt{\frac{g}{\ell}}$ (which is the period of small amplitude pendulum oscillations), we see that the Hamiltonian of the swing spring on phase space $T^{*} \mathbb{R}^{3}$ with canonical coordinates $\left(x, y, z, p_{x}, p_{y}, p_{z}\right)$ is

$$
\widetilde{H}=\frac{1}{2}\left(p_{x}^{2}+p_{y}^{2}+p_{z}^{2}\right)+\widetilde{U}(x, y, z)
$$


where

$$
\widetilde{U}(x, y, z)=z+\frac{1}{2} \nu^{2}\left(1-\frac{1}{\nu^{2}}-\sqrt{x^{2}+y^{2}+z^{2}}\right)^{2}
$$

and $\nu=\sqrt{\frac{k \ell}{m g}}=\sqrt{\frac{\ell}{\ell-\ell_{0}}}$. Since $\ell>\ell_{0}$, we have $\nu>1$. This says that the frequency of the spring oscillation is greater than the frequency of the small amplitude pendulum oscillations. The reason for this is that the frequencies are not independent because they are coupled by (5). Expanding the potential $\widetilde{U}(6)$ about its stable equilibrium $(0,0,-1)$ to cubic terms gives

$$
\widetilde{U}(x, y, z)=\frac{1}{2}\left(x^{2}+y^{2}+z^{2}\right)-\mu\left(x^{2}+y^{2}\right) z,
$$

where $z$ is the displacement from -1 and $\mu=\frac{1}{2}\left(\nu^{2}-1\right)>0$. Thus the cubic approximation to the Hamiltonian of the swing spring in dimensionless variables with its equilibrium shifted to the origin is the Hamiltonian

$$
H^{\nu}=\frac{1}{2}\left(p_{x}^{2}+p_{y}^{2}+p_{z}^{2}\right)+\frac{1}{2}\left(x^{2}+y^{2}+\nu^{2} z^{2}\right)-\mu\left(x^{2}+y^{2}\right) z
$$

on phase space $T^{*} \mathbb{R}^{3}$ with canonical coordinates $Z=\left(x, y, z, p_{x}, p_{y}, p_{z}\right)$. By rescaling the coordinates and changing the time scale, we may consider $\mu$ to be a small parameter, which measures the distance to the origin.

The swing spring Hamiltonian undergoes a $1: 1: 2$ resonance when $\nu=2$. In the remainder of this paper we will study only this case. The Hamiltonian of the cubic approximation of the resonant swing spring is

$$
\mathcal{H}=H_{0}+V=\frac{1}{2}\left(p_{x}^{2}+p_{y}^{2}+p_{z}^{2}\right)+\frac{1}{2}\left(x^{2}+y^{2}+4 z^{2}\right)-\mu\left(x^{2}+y^{2}\right) z .
$$

\section{An approximating integrable system}

In this section we find an integrable approximation to the resonant swing spring $\left(\mathcal{H}, T^{*} \mathbb{R}^{3}, \omega=\mathrm{d} x \wedge \mathrm{d} p_{x}+\mathrm{d} y \wedge \mathrm{d} p_{y}+\mathrm{d} z \wedge \mathrm{d} p_{z}\right)$ by averaging over the flow of the quadratic part of $\mathcal{H}$. Due to the resonance there will be secular terms at cubic order. From general results it is known that a large measure of initial conditions of the averaged system stays close to the original solutions for long times, see e.g. [1].

Clearly the Hamiltonian $\mathcal{H}(9)$ is invariant under rotation about the $z$-axis

$$
\varphi:(s, Z) \mapsto \varphi_{s}(Z)=\left(\begin{array}{ccc|ccc}
\cos s & -\sin s & 0 & 0 & 0 & 0 \\
\sin s & \cos s & 0 & 0 & 0 & 0 \\
0 & 0 & 1 & 0 & 0 & 0 \\
\hline 0 & 0 & 0 & \cos s & -\sin s & 0 \\
0 & 0 & 0 & \sin s & \cos s & 0 \\
0 & 0 & 0 & 0 & 0 & 1
\end{array}\right) Z,
$$


which has momentum

$$
L=x p_{y}-y p_{x} .
$$

The quadratic terms $H_{0}$ of the Hamiltonian $\mathcal{H}(9)$ are in $1: 1: 2$ resonance. The flow of the Hamiltonian vector field $X_{H_{0}}$ corresponding to $H_{0}$ generates the oscillator action

$$
\psi:(t, Z) \mapsto \psi_{t}(Z)=\left(\begin{array}{ccc|ccc}
\cos t & 0 & 0 & \sin t & 0 & 0 \\
0 & \cos t & 0 & 0 & \sin t & 0 \\
0 & 0 & \cos 2 t & 0 & 0 & \frac{1}{2} \sin 2 t \\
-\sin t & 0 & 0 & \cos t & 0 & 0 \\
0 & -\sin t & 0 & 0 & \cos t & 0 \\
0 & 0 & -2 \sin 2 t & 0 & 0 & \cos 2 t
\end{array}\right) Z .
$$

To make the oscillator action a symmetry, we average the Hamiltonian $\mathcal{H}$ (9) over the integral curves of $X_{H_{0}}$. We obtain

$$
\begin{aligned}
\overline{\mathcal{H}}(Z) & =\frac{1}{2 \pi} \int_{0}^{2 \pi} \mathcal{H} \circ \psi_{t}(Z) \mathrm{d} t \\
& =H_{0}-\frac{\mu}{8}\left[\left(x p_{x}+y p_{y}\right) p_{z}+\left(x^{2}+y^{2}\right) z-\left(p_{x}^{2}+p_{y}^{2}\right) z\right] .
\end{aligned}
$$

By construction the Hamiltonian $\overline{\mathcal{H}}$ is invariant under the oscillator action (12). Therefore $\left\{\overline{\mathcal{H}}, H_{0}\right\}=0$, where $\{$,$\} is the standard Poisson bracket$ corresponding to $\omega$. It is straightforward to check that $\overline{\mathcal{H}}$ is also invariant under the axial action (10) and hence $\{\overline{\mathcal{H}}, L\}=0$. Since the axial and oscillator actions commute, it follows that $\left\{H_{0}, L\right\}=0$. Consequently, the system $\left(\overline{\mathcal{H}}, H_{0}, L, T^{*} \mathbb{R}^{3}, \omega\right)$ is Liouville integrable.

For later purposes it is useful to study a different, but equivalent Liouville integrable system. This equivalent system is obtained by a linear symplectic transformation that diagonalizes both, $H_{0}$ and $L$. Consider the invertible linear map

$$
\begin{aligned}
\Psi: T^{*} \mathbb{R}^{3} \rightarrow T^{*} \mathbb{R}^{3}:\left(\xi, \eta, \zeta, p_{\xi}, p_{\eta}, p_{\zeta}\right) \mapsto\left(x, y, z, p_{x}, p_{y}, p_{z}\right)= \\
=\frac{1}{\sqrt{2}}\left(p_{\eta}+\xi, p_{\xi}+\eta, \zeta, p_{\xi}-\eta, p_{\eta}-\xi, 2 p_{\zeta}\right) .
\end{aligned}
$$

Then $\Psi$ is symplectic, that is,

$$
\Psi^{*}(\omega)=\mathrm{d} \xi \wedge \mathrm{d} p_{\xi}+\mathrm{d} \eta \wedge \mathrm{d} p_{\eta}+\mathrm{d} \zeta \wedge \mathrm{d} p_{\zeta}=\widehat{\omega} .
$$

Moreover, $\Psi$ diagonalizes the momenta $L(11)$ and $H_{0}(9)$, namely,

$$
\widehat{L}=\Psi^{*}(L)=\frac{1}{2}\left(p_{\eta}^{2}+\eta^{2}\right)-\frac{1}{2}\left(p_{\xi}^{2}+\xi^{2}\right)
$$


and

$$
\widehat{H}_{0}=\Psi^{*}\left(H_{0}\right)=\frac{1}{2}\left(p_{\xi}^{2}+\xi^{2}+p_{\eta}^{2}+\eta^{2}+2\left(p_{\zeta}^{2}+\zeta^{2}\right)\right)
$$

In new coordinates $\Xi=\left(\xi, \eta, \zeta, p_{\xi}, p_{\eta}, p_{\zeta}\right)$ on $\left(T^{*} \mathbb{R}^{3}, \widehat{\omega}\right)$ the averaged Hamiltonian $\overline{\mathcal{H}}(13)$ becomes

$$
\widehat{H}=\Psi^{*}(\overline{\mathcal{H}})=\widehat{H}_{0}+\lambda\left[\left(\xi p_{\zeta}-\zeta p_{\xi}\right) \eta-\left(\xi \zeta+p_{\xi} p_{\zeta}\right) p_{\eta}\right]
$$

where $\lambda=\frac{\mu \sqrt{2}}{8}$. Hamilton's equations for the integral curves of $X_{\widehat{H}}$ are

$$
\begin{array}{rlrl}
\dot{\xi} & =p_{\xi}-\lambda\left(\eta \zeta+p_{\eta} p_{\zeta}\right) & & \dot{p}_{\xi}=-\xi-\lambda\left(\eta p_{\zeta}-\zeta p_{\eta}\right) \\
\dot{\eta}=p_{\eta}-\lambda\left(\xi \zeta+p_{\xi} p_{\zeta}\right) & & \dot{p}_{\eta}=-\eta-\lambda\left(\xi p_{\zeta}-\zeta p_{\xi}\right) \\
\dot{\zeta}=2 p_{\zeta}+\lambda\left(\xi \eta-p_{\xi} p_{\eta}\right) & & \dot{p}_{\zeta}=-2 \zeta+\lambda\left(\xi p_{\eta}+\eta p_{\xi}\right) .
\end{array}
$$

Introduce new momenta

$$
J^{1}=\frac{1}{2}\left(\widehat{H}_{0}-\widehat{L}\right)=\frac{1}{2}\left(p_{\xi}^{2}+\xi^{2}+p_{\zeta}^{2}+\zeta^{2}\right)
$$

and

$$
J^{2}=\frac{1}{2}\left(\widehat{H}_{0}+\widehat{L}\right)=\frac{1}{2}\left(p_{\eta}^{2}+\eta^{2}+p_{\zeta}^{2}+\zeta^{2}\right),
$$

whose Hamiltonian vectors fields $X_{J^{1}}$ and $X_{J^{2}}$ have flows giving the $S^{1}$ actions

$$
\varphi_{t}^{J^{1}}:(t, \Xi) \mapsto\left(\begin{array}{ccc|ccc}
\cos t & 0 & 0 & \sin t & 0 & 0 \\
0 & 1 & 0 & 0 & 0 & 0 \\
0 & 0 & \cos t & 0 & 0 & \sin t \\
\hline-\sin t & 0 & 0 & \cos t & 0 & 0 \\
0 & 0 & 0 & 0 & 1 & 0 \\
0 & 0 & -\sin t & 0 & 0 & \cos t
\end{array}\right) \Xi
$$

and

$$
\varphi_{s}^{J^{2}}:(s, \Xi) \mapsto\left(\begin{array}{ccc|ccc}
1 & 0 & 0 & 0 & 0 & 0 \\
0 & \cos s & 0 & 0 & \sin s & 0 \\
0 & 0 & \cos s & 0 & 0 & \sin s \\
\hline 0 & 0 & 0 & 1 & 0 & 0 \\
0 & -\sin s & 0 & 0 & \cos s & 0 \\
0 & 0 & -\sin s & 0 & 0 & \cos s
\end{array}\right) \Xi,
$$

respectively. Since $\left\{J^{1}, J^{2}\right\}=0$, these actions commute. Moreover, they leave the Hamiltonian $\widehat{H}(17)$ invariant. Thus $\left(\widehat{H}, J^{1}, J^{2}, T^{*} \mathbb{R}^{3}, \widehat{\omega}\right)$ is a Liouville integrable system.

Equation (18) is a variant of the 3-wave system, see [5] and the references therein. The crucial difference of (18) to the usual presentation of the 3wave system is that we retain the linear terms. These terms usually are removed by the ansatz $\xi+i p_{\xi}=A \exp (i t)$, etc., which leads to equations in the amplitudes $A$ etc., see [5]. In our treatment we retain the linear terms, because they determine the swing plane to lowest order. Keeping these terms enables us to find the swing plane without invoking the "pattern evocation in shape space" hypothesis, as was done in [5]. 


\section{Reduction to one degree of freedom}

In what follows we study the geometry of the energy momentum map

$$
\mathcal{E} \mathcal{M}: T^{*} \mathbb{R}^{3} \rightarrow \mathbb{R}^{3}: \Xi \mapsto\left(\widehat{H}(\Xi), J^{1}(\Xi), J^{2}(\Xi)\right)
$$

of the Liouville integrable system $\left(\widehat{H}, J^{1}, J^{2}, T^{*} \mathbb{R}^{3}, \widehat{\omega}\right)$. This energy momentum map is related to the energy momentum map

$$
\widetilde{\mathcal{E M}}: T^{*} \mathbb{R}^{3} \rightarrow \mathbb{R}^{3}: Z \mapsto\left(\overline{\mathcal{H}}(Z), L(Z), H_{0}(Z)\right)
$$

of the resonant swing spring system by $\mathcal{E} \mathcal{M}=\Lambda \circ \widetilde{\mathcal{E M}} \circ \Psi$. Here $\Psi$ is the linear symplectic map (14) and $\Lambda$ is the invertible linear map $\frac{1}{2}\left(\begin{array}{rrr}2 & 0 & 0 \\ 0 & -1 & 1 \\ 0 & 1 & 1\end{array}\right)$. Thus the Liouville integrable systems $\left(\overline{\mathcal{H}}, L, H_{0}, T^{*} \mathbb{R}^{3}, \omega\right)$ and $\left(\widehat{H}, J^{1}, J^{2}, T^{*} \mathbb{R}^{3}, \widehat{\omega}\right)$ are equivalent.

The simplest way to reduce $\left(\widehat{H}, J^{1}, J^{2}, T^{*} \mathbb{R}^{3}, \widehat{\omega}\right)$ is to consider the twotorus action generated by the momenta $J^{1}$ and $J^{2}$. Concretely, define a $T^{2}$-action

$$
\begin{aligned}
\Phi: T^{2} \times T^{*} \mathbb{R}^{3} & \rightarrow T^{*} \mathbb{R}^{3}:((t, s), \Xi) \mapsto \varphi_{t}^{J^{1}} \circ \varphi_{s}^{J^{2}}(\Xi)= \\
& =\left(\begin{array}{ccc|ccc}
\cos t & 0 & 0 & \sin t & 0 & 0 \\
0 & \cos s & 0 & 0 & \sin s & 0 \\
0 & 0 & \cos (t+s) & 0 & 0 & \sin (t+s) \\
\hline-\sin t & 0 & 0 & \cos t & 0 & 0 \\
0 & -\sin s & 0 & 0 & \cos s & 0 \\
0 & 0 & -\sin (t+s) & 0 & 0 & \cos (t+s)
\end{array}\right) \Xi,
\end{aligned}
$$

which comes from combining the $S^{1}$ actions (21) and (22). From their momentum maps we obtain the momentum mapping

$$
J: T^{*} \mathbb{R}^{3} \rightarrow \mathbb{R}^{2}: \Xi \mapsto\left(J^{1}(\Xi), J^{2}(\Xi)\right)
$$

of $\Phi$. To reduce the integrable system $\left(\widehat{H}, J^{1}, J^{2}, T^{*} \mathbb{R}^{3}, \widehat{\omega}\right)$ by the symmetry $\Phi$, we use invariant theory. In this approach, the generators of the algebra of invariant polynomials are used as new coordinates. The algebra of $T^{2}$ invariant polynomials is generated by

$$
\rho_{1}=p_{\xi}^{2}+\xi^{2}, \quad \rho_{2}=p_{\eta}^{2}+\eta^{2}, \quad \rho_{6}=p_{\zeta}^{2}+\zeta^{2}
$$

and

$$
\rho_{4}=\left(\xi \eta-p_{\xi} p_{\eta}\right) p_{\zeta}-\left(\xi p_{\eta}+\eta p_{\xi}\right) \zeta, \quad \rho_{5}=\left(\xi \eta-p_{\xi} p_{\eta}\right) \zeta+\left(\xi p_{\eta}+\eta p_{\xi}\right) p_{\zeta}
$$


The invariance of $\rho_{4}$ and $\rho_{5}$ is obvious if we write them as

$$
\rho_{5}+i \rho_{4}=\left(\xi-i p_{\xi}\right)\left(\eta-i p_{\eta}\right)\left(\zeta+i p_{\zeta}\right),
$$

Note that the cubic part of $\widehat{H}$ is exactly $\lambda \rho_{4}$. The invariants are subject to the relation

$$
\rho_{4}^{2}+\rho_{5}^{2}=\rho_{1} \rho_{2} \rho_{6}, \quad \rho_{1} \geq 0, \rho_{2} \geq 0, \rho_{6} \geq 0 .
$$

Therefore the space $P_{j_{1}, j_{2}}=J^{-1}\left(j_{1}, j_{2}\right) / T^{2}$ of orbits of $\Phi$ with momentum $\left(j_{1}, j_{2}\right)$ is defined by $(28)$ together with

$$
\rho_{1}+\rho_{6}=2 j_{1}, \quad \rho_{2}+\rho_{6}=2 j_{2},
$$

which just expresses $J^{1}$ and $J^{2}$ in terms of invariants. Eliminating $\rho_{1}$ and $\rho_{2}$ gives

$$
G\left(\rho_{4}, \rho_{5}, \rho_{6}\right)=\rho_{4}^{2}+\rho_{5}^{2}-\rho_{6}\left(2 j_{1}-\rho_{6}\right)\left(2 j_{2}-\rho_{6}\right)=0,
$$

where $0 \leq \rho_{6} \leq \min \left(2 j_{1}, 2 j_{2}\right)$. This defines the fully reduced space $P_{j_{1}, j_{2}}$ as a submanifold of $\mathbb{R}^{3}$ with coordinates $\left(\rho_{4}, \rho_{5}, \rho_{6}\right)$. Because of the restriction on $\rho_{6}$ and since $\rho_{4}^{2}+\rho_{5}^{2}$ is nonnegative, there are essentially three possibilities for the geometry of $P_{j_{1}, j_{2}}$, see Figure 1. These possibilities are determined by the position of the roots of the polynomial

$$
P_{3}\left(\rho_{6}\right)=\rho_{6}\left(2 j_{1}-\rho_{6}\right)\left(2 j_{2}-\rho_{6}\right) .
$$

1. $j_{1} \neq j_{2} \neq 0$. The space $J^{-1}\left(j_{1}, j_{2}\right)$ is smooth and the action $\Phi$ is free. Then the fully reduced space is either

$$
P_{j_{1}, j_{2}}^{>}: \rho_{4}^{2}+\rho_{5}^{2}=\rho_{6}\left(2 j_{1}-\rho_{6}\right)\left(2 j_{2}-\rho_{6}\right), \quad j_{1}>j_{2}>0, \quad 0 \leq \rho_{6} \leq 2 j_{2},
$$

or

$$
P_{j_{1}, j_{2}}^{<}: \rho_{4}^{2}+\rho_{5}^{2}=\rho_{6}\left(2 j_{1}-\rho_{6}\right)\left(2 j_{2}-\rho_{6}\right), \quad j_{2}>j_{1}>0, \quad 0 \leq \rho_{6} \leq 2 j_{1},
$$

which are each diffeomorphic to a smooth 2-sphere. Every point on the fully reduced space reconstructs to a two-torus orbit of the free action $\Phi$ in phase space.

2. $j_{1}=j_{2} \neq 0$. The space $J^{-1}\left(j_{1}, j_{1}\right)$ is smooth and the action $\Phi$ has a fixed point $\left(\rho_{4}, \rho_{5}, \rho_{6}\right)=\left(0,0,2 j_{1}\right)$ with isotropy group $S^{1}$. Then the fully reduced space

$$
P_{j_{1}, j_{1}}: \rho_{4}^{2}+\rho_{5}^{2}=\rho_{6}\left(2 j_{1}-\rho_{6}\right)^{2}, \quad j_{1}>0,0 \leq \rho_{6} \leq 2 j_{1},
$$



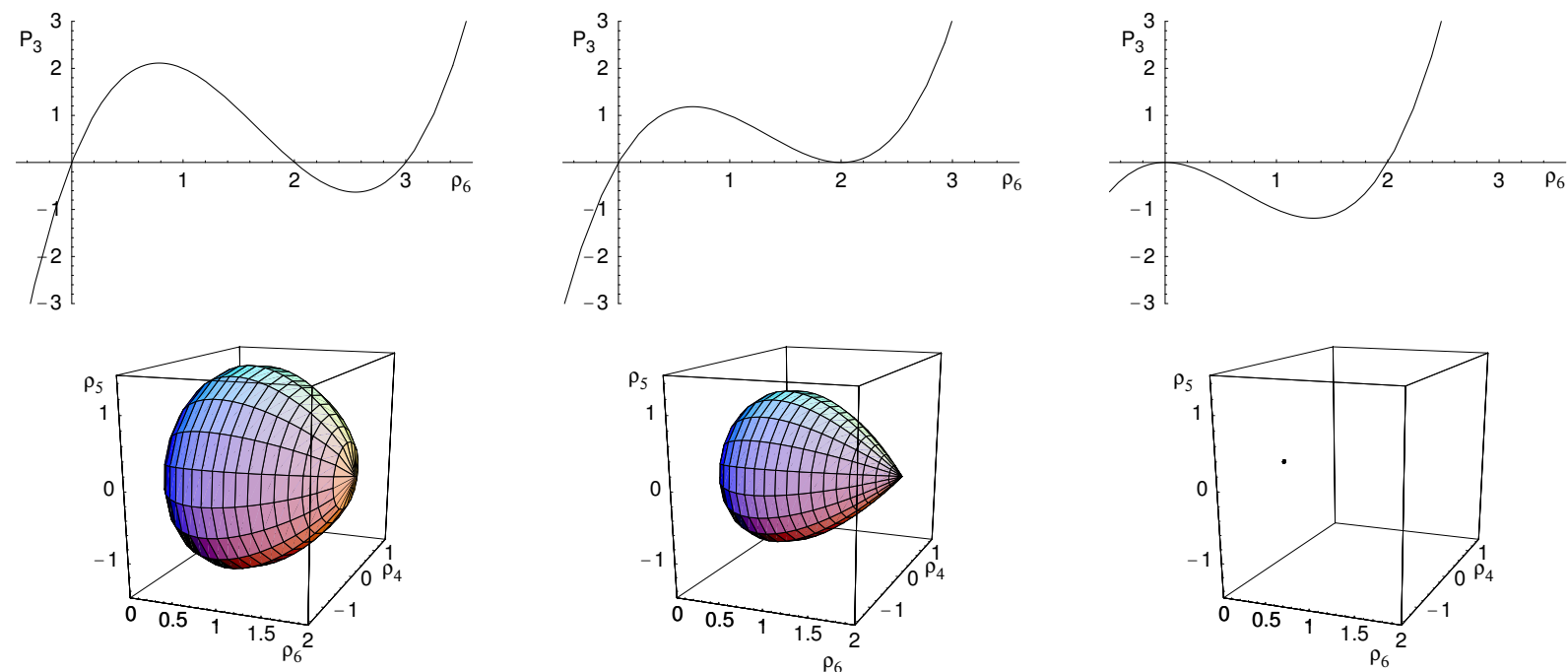

Figure 1: The polynomial $P_{3}$ (upper row) and the corresponding reduced spaces $P_{j_{1}, j_{2}}$ (lower row) illustrating the three types of reduced space using $j_{1}=1, j_{2}=3 / 2$ (left), $j_{1}=j_{2}=1$ (middle), and $j_{1}=0, j_{2}=1$ (right).

is a topological 2-sphere with one (conical) singular point $\left(0,0,2 j_{1}\right)$. This singular point reconstructs to a pure springing motion on $\xi=\eta=p_{\xi}=p_{\eta}=0$ and $\zeta^{2}+p_{\zeta}^{2}=2 j_{1}$. The general point of the fully reduced space reconstructs to a two-torus in phase space which has angular momentum zero. This twotorus dynamically decomposes into an $S^{1}$ family of planar $S^{1}$ motions.

3. $j_{1}=0$ or $j_{2}=0$. The fully reduced spaces $P_{j_{1}, 0}, j_{1}>0$ and $P_{0, j_{2}}$, $j_{2}>0$ each are a point $\rho_{4}=\rho_{5}=\rho_{6}=0$. In $T^{*} \mathbb{R}^{3}$ this reconstructs to a pure swinging motion with nonzero angular momentum on the circle $\xi^{2}+p_{\xi}^{2}=2 j_{1}$, $\eta=p_{\eta}=\zeta=p_{\zeta}=0$ (counterclockwise in the $(x, y)$ plane projection) or the circle $\eta^{2}+p_{\eta}^{2}=2 j_{2}, \xi=p_{\xi}=\zeta=p_{\zeta}=0$ (clockwise), respectively. If $j_{1}=j_{2}=0$, then $P_{0,0}$ is the point $\rho_{2}=\rho_{5}=\rho_{6}=0$, which reconstructs to the equilibrium point $\xi=p_{\xi}=\eta=p_{\eta}=\zeta=p_{\zeta}=0$.

Using physical language, one would say that the projection of a generic motion of the resonant swing spring to the $(x, y)$ plane is elliptically, linearly, or circularly polarized, corresponding to cases $1,2,3$, respectively.

Because the Hamiltonian $\widehat{H}(17)$ is invariant under the $T^{2}$ action $\Phi$, it 


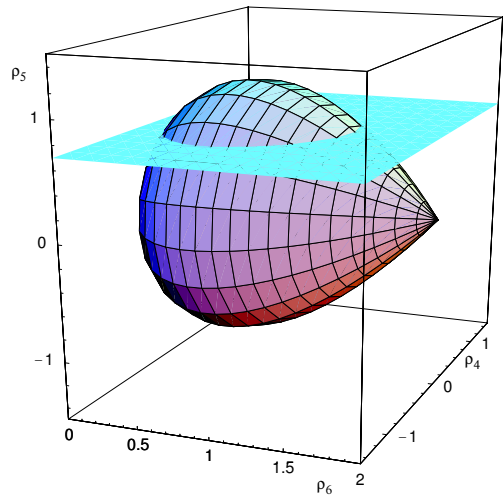

Figure 2: The intersection of the singular reduced space $P_{1,1}$ with the plane $h=$ const gives an integral curves of the reduced one degree of freedom system.

induces the fully reduced Hamiltonian

$$
H_{j_{1}, j_{2}}: P_{j_{1}, j_{2}} \subseteq \mathbb{R}^{3} \rightarrow \mathbb{R}:\left(\rho_{4}, \rho_{5}, \rho_{6}\right) \mapsto j_{1}+j_{2}+\lambda \rho_{4}
$$

The integral curves of the reduced system are now given by the intersection of the reduced phase spaces $\{G=0\}$ and the planes $\left\{H_{j_{1}, j_{2}}=h\right\}$, as illustrated in Figure 2. The fact that the Hamiltonian is a linear function does not imply that the system is a linear dynamical system, because the nonlinearity is contained in the Poisson bracket that gives the dynamics on the reduced space. A direct calculation shows that on $C^{\infty}\left(\mathbb{R}^{3}\right)$, where $\mathbb{R}^{3}$ has coordinates $\left(\rho_{4}, \rho_{5}, \rho_{6}\right)$, the original canonical Poisson bracket on phase space $\left(T \mathbb{R}^{3}, \omega\right)$ induces a Poisson bracket $\{$,$\} on \mathbb{R}^{6}$ with coordinates $\left(\rho_{1}, \ldots, \rho_{6}\right)$. Its nonzero brackets are given by

$$
\begin{aligned}
& \left\{\rho_{1}, \rho_{4}\right\}=\left\{\rho_{2}, \rho_{4}\right\}=\left\{\rho_{4}, \rho_{6}\right\}=-2 \rho_{5}, \\
& \left\{\rho_{1}, \rho_{5}\right\}=\left\{\rho_{2}, \rho_{5}\right\}=\left\{\rho_{5}, \rho_{6}\right\}=2 \rho_{4}, \\
& \left\{\rho_{4}, \rho_{5}\right\}=\left(\rho_{1}+\rho_{2}\right) \rho_{6}-\rho_{1} \rho_{2} .
\end{aligned}
$$

These brackets have the generators of the symmetry $\rho_{1}+\rho_{6}$ and $\rho_{2}+\rho_{6}$ as Casimirs, which we then use to eliminate $\rho_{1}$ and $\rho_{2}$. This shows that a Poisson bracket on the fully reduced space $P_{j_{1}, j_{2}}$ (considered as a subset of 
$\mathbb{R}^{3}$ with coordinates $\left.\left(\rho_{4}, \rho_{5}, \rho_{6}\right)\right)$ is

$$
\begin{aligned}
& \left\{\rho_{6}, \rho_{4}\right\}=\frac{\partial G}{\partial \rho_{5}}=2 \rho_{5} \\
& \left\{\rho_{4}, \rho_{5}\right\}=\frac{\partial G}{\partial \rho_{6}}=\rho_{6}\left(2 j_{1}+2 j_{2}-\rho_{6}\right)-\left(2 j_{1}-\rho_{6}\right)\left(2 j_{2}-\rho_{6}\right) \\
& \left\{\rho_{5}, \rho_{6}\right\}=\frac{\partial G}{\partial \rho_{4}}=2 \rho_{4},
\end{aligned}
$$

where $G$ is given in (30).

Hence the integral curves of the fully reduced Hamiltonian vector field $X_{H_{j_{1}, j_{2}}}$ on $\left(P_{j_{1}, j_{2}},\{\},\right)$ satisfy

$$
\begin{aligned}
& \dot{\rho}_{4}=\left\{\rho_{4}, H_{j_{1}, j_{2}}\right\}=0 \\
& \dot{\rho}_{5}=\left\{\rho_{5}, H_{j_{1}, j_{2}}\right\}=\lambda P_{3}^{\prime}\left(\rho_{6}\right), \quad \text { see }(31) \\
& \dot{\rho}_{6}=\left\{\rho_{6}, H_{j_{1}, j_{2}}\right\}=2 \lambda \rho_{5}
\end{aligned}
$$

When $j_{1}=j_{2}>0$, the $2 j_{1}$-level set of the fully reduced Hamiltonian $H_{j_{1}, j_{1}}$ is the intersection of the 2-plane $\left\{\rho_{4}=0\right\}$ with the fully reduced space $P_{j_{1}, j_{1}}$. This level set is an orbit of $X_{H_{j_{1}, j_{1}}}$, which is homoclinic to the conical singular point $\left(0,0,2 j_{1}\right)$ of $P_{j_{1}, j_{1}}$. The conical singular point reconstructs to the hyperbolic periodic orbit $\gamma$ of pure springing motion; while the homoclinic loop reconstructs to a 2 -torus bundle over each point of $H_{j_{1}, j_{1}}^{-1}\left(2 j_{1}\right) \backslash\left\{\left(0,0,2 j_{1}\right)\right\}$. Thus $H_{j_{1}, j_{1}}^{-1}\left(2 j_{1}\right)$ reconstructs to the stable and unstable manifold in $T^{*} \mathbb{R}^{3}$ of the hyperbolic periodic orbit $\gamma$. This invariant manifold is topologically the product of a once pinched 2-torus and a circle.

Describing the geometry of the 3-tori and the dynamics of the swing spring near this homoclinic invariant manifold is the main objective of the remainder of this paper.

\section{The critical values of $\mathcal{E M}$}

We now determine the set of critical values of the energy momentum mapping $\mathcal{E M}(23)$ of the Liouville integrable system $\left(\widehat{H}, J^{1}, J^{2}, T^{*} \mathbb{R}^{3}, \widehat{\omega}\right)$. The set of critical values of $\mathcal{E M}$ is called bifurcation diagram $\Sigma$. The critical values of $\mathcal{E} \mathcal{M}$ can be are determined from the equilibrium points of the reduced system. 


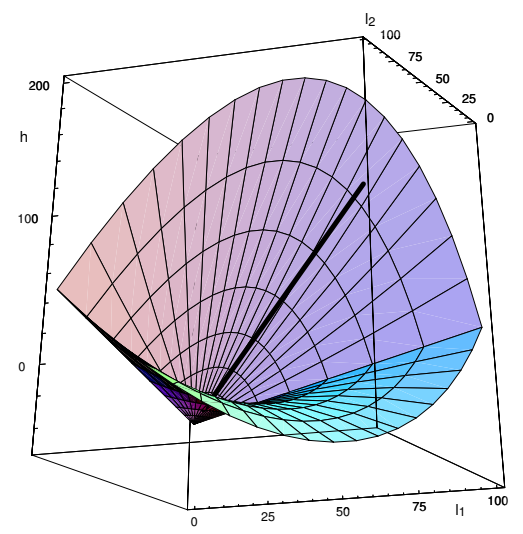

Figure 3: Set of critical values of the energy momentum map (bifurcation diagram). It consists of two smooth patches intersecting in two lines and a thread (thick black line) connected to the former only at the origin. The image of the momentum map contains the thread, hence it is not simply connected.

Geometrically this occurs for energies $h$ for which the plane $\left\{H_{j_{1}, j_{2}}=h\right\}$ intersects the fully reduced space $P_{j_{1}, j_{2}}$ in a (singular) point. This occurs in different ways for the three types of reduced spaces. In case 1, it occurs through a tangency of $\left\{H_{j_{1}, j_{2}}=h\right\}$ with $P_{j_{1}, j_{2}}$. In case 2 , it is either again a tangency or the plane $\left\{H_{j_{1}, j_{2}}=h\right\}$ contains the conical singular point $\left(0,0,2 j_{1}\right)$. Hence $\left(j_{1}, j_{2}, h\right)=\left(j_{1}, j_{1}, 2 j_{1}\right)$. In case 3 , either $j_{1}$ or $j_{2}$ is zero, so that the critical values are $\left(j_{1}, 0, j_{1}\right)$ or $\left(0, j_{2}, j_{2}\right)$, respectively.

The points of tangency between $\left\{H_{j_{1}, j_{2}}=h\right\}$ and $P_{j_{1}, j_{2}}$ (30) can be obtained using Lagrange multipliers. This leads to the condition that the polynomial

$$
Q\left(\rho_{6}\right)=-\frac{1}{\lambda^{2}}\left(j_{1}+j_{2}-h\right)^{2}+\rho_{6}\left(2 j_{1}-\rho_{6}\right)\left(2 j_{2}-\rho_{6}\right)
$$

has a multiple zero in $\left[0, \min \left(2 j_{1}, 2 j_{2}\right)\right]$. When $j_{1}=j_{2} \geq 0$ and $h=2 j_{1}$, we see that $2 j_{1}$ is a multiple root of $Q$ in $\left[0,2 j_{1}\right]$.

The following argument determines a parametrization of the bifurcation diagram $\Sigma$. The bifurcation diagram $\Sigma$ is contained in the set of $\left(h, j_{1}, j_{2}\right) \in$ $\mathbb{R}^{3}$ where the polynomial $Q(36)$ has a multiple complex root. This set is the discriminant locus of the polynomial $Q$. If we restrict ourselves to the set of $\left(h, j_{1}, j_{2}\right) \in \mathbb{R}^{3}$ where the polynomial $Q$ (36) has a multiple root in $\left[0, \min \left(2 j_{1}, 2 j_{2}\right)\right]$, then we obtain the restricted discriminant locus $\Delta$ of $Q$, 
which is equal the set of critical values $\Sigma$ of the energy momentum mapping $\mathcal{E} \mathcal{M}$. When $\left(h, j_{1}, j_{2}\right) \in \Delta$, then $Q$ may be written as

$$
\begin{aligned}
Q\left(\rho_{6}\right) & =\rho_{6}^{3}-2\left(j_{1}+j_{2}\right) \rho_{6}^{2}+4 j_{1} j_{2} \rho_{6}-\frac{1}{\lambda^{2}}\left(j_{1}+j_{2}-h\right)^{2} \\
& =\left(\rho_{6}-s\right)^{2}\left(\rho_{6}-t\right), \quad s \in\left[0, \min \left(2 j_{1}, 2 j_{2}\right)\right] \\
& =\rho_{6}^{3}-(t+2 s) \rho_{6}^{2}+\left(2 s t+s^{2}\right) \rho_{6}-s^{2} t .
\end{aligned}
$$

Comparing coefficients gives

$$
\begin{aligned}
t+2 s & =2\left(j_{1}+j_{2}\right) \\
2 s t+s^{2} & =4 j_{1} j_{2} \\
s^{2} t & =\frac{1}{\lambda^{2}}\left(j_{1}+j_{2}-h\right)^{2} .
\end{aligned}
$$

Since $s \geq 0$, from the third equation in (37) it follows that $t \geq 0$. Taking the square root of both sides of this equation gives

$$
j_{1}+j_{2}-h=\varepsilon_{1} \lambda s \sqrt{t}
$$

where $\varepsilon_{1}= \pm$. Using the first two equations in (37), we see that

$$
\begin{aligned}
4\left(j_{1}-j_{2}\right)^{2} & =4\left(j_{1}+j_{2}\right)^{2}-16 j_{1} j_{2} \\
& =(t+2 s)^{2}-4\left(2 s t+s^{2}\right)=t(t-4 s)
\end{aligned}
$$

which implies that

$$
\text { either } t \geq 4 s \text { and } t \geq 0 \text {, or } t=0 \text {. }
$$

When either of the conditions (40) hold, we may take the square root of both sides of (39). We then obtain

$$
j_{1}-j_{2}=\frac{1}{2} \varepsilon_{2} \sqrt{t(t-4 s)},
$$

where $\varepsilon_{2}= \pm$. Adding and subtracting (38) from

$$
j_{1}+j_{2}=\frac{1}{2}(t+2 s)
$$

(see the first equation in (37)) gives

$$
\begin{aligned}
& j_{1}=j_{1}(s, t)=\frac{1}{4}\left(t+2 s+\varepsilon_{2} \sqrt{t(t-4 s)}\right) \\
& j_{2}=j_{2}(s, t)=\frac{1}{4}\left(t+2 s-\varepsilon_{2} \sqrt{t(t-4 s)}\right) .
\end{aligned}
$$


Subtracting (42) from (38) we obtain

$$
h=h(s, t)=\frac{1}{2}(t+2 s)-\varepsilon_{1} \lambda s \sqrt{t} .
$$

Consider the mapping

$$
\mathcal{P}_{\varepsilon_{1}, \varepsilon_{2}}: D \subseteq \mathbb{R}^{2} \rightarrow \mathbb{R}^{3}:(s, t) \mapsto\left(h(s, t), j_{1}(s, t), j_{2}(s, t)\right),
$$

where $D$ is the closed subset of $\mathbb{R}^{2}$ which is the union of the intersection of the closed half planes $\{s \geq 0\},\{t \geq 0\},\{t-4 s \geq 0\}$ and the closed half line $L_{-}=\left\{(s, 0) \in \mathbb{R}^{2} \mid s \geq 0\right\}$. For every choice of sign of $\varepsilon_{1}$ and $\varepsilon_{2}$, the map $\mathcal{P}_{\varepsilon_{1}, \varepsilon_{2}}$ parametrizes a patch of the bifurcation diagram $\Sigma$. All the patches together are shown in Figure 3.

The image under $\mathcal{P}_{\varepsilon_{1}, \varepsilon_{2}}$ of the closed half line $L_{-}$is the thread $\mathcal{T}=$ $\left\{\left(2 j_{1}, j_{1}, j_{1}\right) \in \mathbb{R}^{3} \mid j_{1} \geq 0\right\}$, which is attached to the 2-dimensional pieces $\mathcal{P}_{\varepsilon_{1}, \varepsilon_{2}}\left(D \backslash L_{-}\right)$of $\Sigma$ only at the origin $(0,0,0)$. Since the range $\mathcal{I}$ of the energy momentum mapping $\mathcal{E} \mathcal{M}(23)$ is the closed region in $\mathbb{R}^{3}$ bounded by $\Sigma$ and containing the thread $\mathcal{T}$, we see that the set of regular values $\mathcal{I} \backslash \mathcal{T}$ in the image of $\mathcal{E} \mathcal{M}$ is not simply connected. Thus the Liouville integrable system $\left(\widehat{H}, J^{1}, J^{2}, T^{*} \mathbb{R}^{3}, \widehat{\omega}\right)$ possibly can have monodromy. The thread $\mathcal{T}$ represents the unstable springing motion including its separatrix. The intersections of two patches, which form 2-dimensional pieces in the boundary of the image $\mathcal{I}$, represent the periodic orbits of left or right circular swinging motion. Any other point on the boundary represents two-tori. Their special feature is that the instantaneous ellipse in the $(x, y)$-plane formed by their projection has constant excentricity, see section 8 below. All the remaining points in the image of $\mathcal{E} \mathcal{M}$ represent generic motion on three-tori.

For the geometrically inclined reader, we will show in sections 6 and 7 that the integrable system $\left(\widehat{H}, J^{1}, J^{2}, T \mathbb{R}^{3}, \widehat{\omega}\right)$ has monodromy. Analysts may skip these sections on the first reading and go directly to the derivation of the swing plane angle in section 8 . This provides an analytic proof of the fact that the resonant swing spring has monodromy.

\section{A different reduction}

To uncover the geometry of the energy momentum map $\mathcal{E} \mathcal{M}(23)$, we reduce the Liouville integrable system $\left(\widehat{H}, J^{1}, J^{2}, T^{*} \mathbb{R}^{3}, \widehat{\omega}\right)$ to a two degree of freedom Hamiltonian system by removing the $S^{1}$-symmetry $\varphi_{t}^{J^{1}}(21)$. As removing 
the symmetry induced by $\varphi_{s}^{J^{2}}(22)$ leads to the one degree of freedom system we have already discussed in section 4 , we will omit this second reduction.

To reduce the $S^{1}$-symmetry $\varphi_{t}^{J^{1}}$ we use invariant theory. The algebra of polynomials on $T^{*} \mathbb{R}^{3}$, which is invariant under this symmetry, is generated by

$$
\begin{array}{rlrlrl}
\sigma_{1} & =p_{\xi}^{2}+\xi^{2} & \sigma_{3} & =\eta & \sigma_{5} & =\xi p_{\zeta}-\zeta p_{\xi} \\
\sigma_{2}=p_{\zeta}^{2}+\zeta^{2} & \sigma_{4} & =p_{\eta} & \sigma_{6} & =\xi \zeta+p_{\xi} p_{\zeta} .
\end{array}
$$

These invariants are subject to the relation

$$
\sigma_{5}^{2}+\sigma_{6}^{2}=\sigma_{1} \sigma_{2}, \sigma_{1} \geq 0, \sigma_{2} \geq 0,
$$

which define the space $T^{*} \mathbb{R}^{3} / S^{1}$ of orbits of the $S^{1}$-action $\varphi_{t}^{J^{1}}$. The reduced space $P_{j_{1}}=\left(J^{1}\right)^{-1}\left(j_{1}\right) / S^{1}$ of orbits of $\varphi^{J^{1}}$ on the $j_{1}$-level set of the momentum $J^{1}(19)$ is defined by (46) together with

$$
\frac{1}{2}\left(\sigma_{1}+\sigma_{2}\right)=j_{1} \geq 0 .
$$

Eliminating $\sigma_{1}$ from (46) using (47) gives

$$
\sigma_{5}^{2}+\sigma_{6}^{2}=\sigma_{2}\left(2 j_{1}-\sigma_{2}\right), \quad 0 \leq \sigma_{2} \leq 2 j_{1},
$$

which defines $P_{j_{1}}$ as a submanifold of $\mathbb{R}^{5}$ (with coordinates $\left(\sigma_{2}, \ldots, \sigma_{6}\right)$ ). Since the Hamiltonian $\widehat{H}(17)$ is invariant under $\varphi_{t}^{J^{1}}$, it induces the Hamiltonian

$$
\widehat{H}_{j_{1}}: P_{j_{1}} \rightarrow \mathbb{R}:\left(\sigma_{2}, \sigma_{3}, \ldots, \sigma_{6}\right) \mapsto j_{1}+\frac{1}{2}\left(\sigma_{2}+\sigma_{3}^{2}+\sigma_{4}^{2}\right)+\lambda\left(\sigma_{3} \sigma_{5}-\sigma_{4} \sigma_{6}\right),
$$

where $\lambda=\frac{\mu \sqrt{2}}{8}$.

Consider $\mathbb{R}^{6}$ with coordinates $\left(\sigma_{1}, \sigma_{2}, \ldots, \sigma_{6}\right)$. On $C^{\infty}\left(\mathbb{R}^{6}\right)$ there is a Poisson bracket $\{$,$\} , induced from the Poisson bracket on the space of smooth$ functions on $\left(T^{*} \mathbb{R}^{3}, \widehat{\omega}\right)$, such that the nonzero brackets are

$$
\begin{array}{lll}
\left\{\sigma_{5}, \sigma_{1}\right\}=2 \sigma_{6} & \left\{\sigma_{6}, \sigma_{1}\right\}=-2 \sigma_{5} & \left\{\sigma_{5}, \sigma_{2}\right\}=-2 \sigma_{6} \\
\left\{\sigma_{6}, \sigma_{2}\right\}=2 \sigma_{5} & \left\{\sigma_{4}, \sigma_{3}\right\}=-1 & \left\{\sigma_{6}, \sigma_{5}\right\}=\sigma_{1}-\sigma_{2} .
\end{array}
$$

Note that $\sigma_{1}+\sigma_{2}$ and $\sigma_{5}^{2}+\sigma_{6}^{2}-\sigma_{1} \sigma_{2}$ are Casimirs for the Poisson algebra $\left(C^{\infty}\left(\mathbb{R}^{6}\right),\{\},, \cdot\right)$. The reduced Hamiltonian vector field $X_{\widehat{H}_{j_{1}}}$ on $\mathbb{R}^{6}$ has integral curves which satisfy

$$
\begin{array}{ll}
\dot{\sigma}_{1}=-2 \lambda\left(\sigma_{3} \sigma_{6}+\sigma_{4} \sigma_{5}\right) & \dot{\sigma}_{4}=-\sigma_{3}-\lambda \sigma_{5} \\
\dot{\sigma}_{2}=2 \lambda\left(\sigma_{3} \sigma_{6}+\sigma_{4} \sigma_{5}\right) & \dot{\sigma}_{5}=-\sigma_{6}+\lambda \sigma_{4}\left(\sigma_{1}-\sigma_{2}\right) \\
\dot{\sigma}_{3}=\sigma_{4}-\lambda \sigma_{6} & \dot{\sigma}_{6}=\sigma_{5}+\lambda \sigma_{3}\left(\sigma_{1}-\sigma_{2}\right) .
\end{array}
$$




\section{Monodromy}

To show that the Liouville integrable system $\left(\widehat{H}, J^{1}, J^{2}, T^{*} \mathbb{R}^{3}, \widehat{\omega}\right)$ has monodromy, we fix $j_{1}>0$ and reduce the $S^{1}$ action $\varphi_{t}^{J^{1}}(21)$. We obtain the two degree of freedom Liouville integrable system $\left(\widehat{H}_{j_{1}}, \widehat{J}^{2}, P_{j_{1}},\{\},\right)$, where $\widehat{J}^{2}=\frac{1}{2}\left(\sigma_{2}+\sigma_{3}^{2}+\sigma_{4}^{2}\right)$ is induced from $J^{2}(22)$. We will show that this system satisfies the hypotheses of the monodromy theorem as stated in Cushman and Duistermaat, (see also Matveev [9] and Zung [12]). Thus it has monodromy. This shows that the original Liouville integrable system also has monodromy.

First, we verify that the reduced space $P_{j_{1}}, j_{1}>0$ is a smooth manifold. Since $P_{j_{1}} \subseteq \mathbb{R}^{5}$ is defined by

$$
F(\widetilde{\sigma})=\sigma_{5}^{2}+\sigma_{6}^{2}-2 j_{1} \sigma_{2}+\sigma_{2}^{2}=0,0 \leq \sigma_{2} \leq 2 j_{1},
$$

the point $\widetilde{\sigma}=\left(\sigma_{2}, \ldots, \sigma_{6}\right)$ is singular if

$$
(0,0,0,0,0)=D F(\widetilde{\sigma})=\left(2\left(\sigma_{2}-j_{1}\right), 0,0,2 \sigma_{5}, 2 \sigma_{6}\right)
$$

that is, $\sigma_{2}=j_{1}$ and $\sigma_{5}=\sigma_{6}=0$. But $F\left(j_{1}, \sigma_{3}, \sigma_{4}, 0,0\right)=-j_{1}^{2} \neq 0$. Therefore $P_{j_{1}}$ has no singular points.

Second, observe that the $S^{1}$ action on $P_{j_{1}}$ induced by the $S^{1}$ action $\varphi_{s}^{J^{2}}$ $(22)$ is

$$
\varphi_{s}^{\widehat{J}^{2}}:(s, \sigma) \mapsto\left(\begin{array}{cc|cc|cc}
1 & 0 & 0 & 0 & 0 & 0 \\
0 & 1 & 0 & 0 & 0 & 0 \\
\hline 0 & 0 & \cos s & -\sin s & 0 & 0 \\
0 & 0 & \sin s & \cos s & 0 & 0 \\
\hline 0 & 0 & 0 & 0 & \cos s & \sin s \\
0 & 0 & 0 & 0 & -\sin s & \cos s
\end{array}\right) \sigma .
$$

The action $\varphi_{s}^{\widehat{J}^{2}}$ has a unique fixed point $\widetilde{\sigma}^{0}=\left(2 j_{1}, 0,0,0,0\right)$ on $P_{j_{1}} \subseteq \mathbb{R}^{5}$. Since the reduced Hamiltonian

$$
\widehat{H}_{j_{1}}=j_{1}+\frac{1}{2}\left(\sigma_{2}+\sigma_{3}^{2}+\sigma_{4}^{2}\right)+\lambda\left(\sigma_{3} \sigma_{5}-\sigma_{4} \sigma_{6}\right)
$$

is invariant under $\varphi_{s}^{\widehat{J}^{2}}$, the point $\widetilde{\sigma}^{0}$ is a critical point of $\widehat{H}_{j_{1}}$. Because $\left\{\widehat{H}_{j_{1}}, \widehat{J}^{2}\right\}=0$, the Hamiltonian vector fields $X_{\widehat{H}_{j_{1}}}$ and $X_{\widehat{J}^{2}}$ commute. From (51) and the fact that $\sigma_{1}+\sigma_{2}=2 j_{1}$, we know that $X_{\widehat{H}_{j_{1}}}$ on $\mathbb{R}^{5}$ is

$$
\begin{aligned}
2 \lambda\left(\sigma_{3} \sigma_{6}\right. & \left.+\sigma_{4} \sigma_{5}\right) \frac{\partial}{\partial \sigma_{2}}+\lambda \sigma_{6} \frac{\partial}{\partial \sigma_{3}}+\lambda \sigma_{5} \frac{\partial}{\partial \sigma_{4}} \\
& +\left(\sigma_{6}-2 \lambda \sigma_{4}\left(j_{1}-\sigma_{2}\right)\right) \frac{\partial}{\partial \sigma_{5}}-\left(\sigma_{5}+2 \lambda \sigma_{3}\left(j_{1}-\sigma_{2}\right)\right) \frac{\partial}{\partial \sigma_{6}}
\end{aligned}
$$


Clearly $X_{\widehat{H}_{j_{1}}}\left(\widetilde{\sigma}^{0}\right)=0$. Moreover, the linearization of $X_{\widehat{H}_{j_{1}}}$ on the tangent space

$$
T_{\widetilde{\sigma}^{0}} P_{j_{1}}=\operatorname{ker} D F\left(\widetilde{\sigma}^{0}\right)=\operatorname{ker}\left(2 j_{1}, 0,0,0,0\right)=\operatorname{span}\left\{\frac{\partial}{\partial \sigma_{3}}, \frac{\partial}{\partial \sigma_{4}}, \frac{\partial}{\partial \sigma_{5}}, \frac{\partial}{\partial \sigma_{6}}\right\}
$$

is

$$
Y=D X_{\widehat{H}_{j_{1}}}\left(\widetilde{\sigma}^{0}\right) \mid T_{\widetilde{\sigma}^{0}} P_{j_{1}}=\left(\begin{array}{cccc}
0 & 0 & 0 & \lambda \\
0 & 0 & \lambda & 0 \\
0 & 2 \lambda j_{1} & 0 & 1 \\
2 \lambda j_{1} & 0 & -1 & 0
\end{array}\right) .
$$

Since the characteristic polynomial of $Y$ is $\left(x-\lambda \sqrt{2 j_{1}}\right)^{2}\left(x+\lambda \sqrt{2 j_{1}}\right)^{2}$, the equilibrium point $\widetilde{\sigma}^{0}$ is hyperbolic.

We now look at the energy momentum mapping

$$
\begin{aligned}
\mathcal{E M}_{j_{1}} & : P_{j_{1}} \subseteq \mathbb{R}^{5} \rightarrow \mathbb{R}^{2}: \widetilde{\sigma} \mapsto\left(\widehat{H}_{j_{1}}(\widetilde{\sigma}), \widehat{J}^{2}(\widetilde{\sigma})\right)= \\
& =\left(j_{1}+\frac{1}{2}\left(\sigma_{2}+\sigma_{3}^{2}+\sigma_{4}^{2}\right)+\lambda\left(\sigma_{3} \sigma_{5}-\sigma_{4} \sigma_{6}\right), \frac{1}{2}\left(\sigma_{2}+\sigma_{3}^{2}+\sigma_{4}^{2}\right)\right)
\end{aligned}
$$

of the Liouville integrable system $\left(\widehat{H}_{j_{1}}, \widehat{J}^{2}, P_{j_{1}},\{\},\right)$ with fixed $j_{1}>0$. The fiber $\mathcal{E M}_{j_{1}}^{-1}\left(h, j_{2}\right)$ is compact. To see this note that $\sigma_{2} \geq 0$ on $P_{j_{1}}$ and $\sigma_{2}+\sigma_{3}^{2}+\sigma_{4}^{2}=j_{2} \geq 0$. Therefore $0 \leq \sigma_{2} \leq j_{2}, 0 \leq \sigma_{3} \leq \sqrt{j_{2}}$ and $0 \leq \sigma_{4} \leq$ $\sqrt{j_{2}}$. But

$$
\sigma_{5}^{2}+\sigma_{6}^{2}=\sigma_{2}\left(2 j_{1}-\sigma_{2}\right), \quad 0 \leq \sigma_{2} \leq 2 j_{1}
$$

is the defining equation of $P_{j_{1}}$. Thus $\sigma_{5}$ and $\sigma_{6}$ are bounded. Hence $\mathcal{E} \mathcal{M}_{j_{1}}^{-1}(h$, $j_{2}$ ) is compact. In other words, $\mathcal{E M}_{j_{1}}$ is a proper map. To show that $\mathcal{E M}_{j_{1}}^{-1}\left(h, j_{2}\right)$ is connected, note that it is the total space of an $S^{1}$ bundle over the $h$-level set of the fully reduced Hamiltonian $H_{j_{1}, j_{2}}(32)$ on the fully reduced space $P_{j_{1}, j_{2}}(25)$. Since this level set is connected, when $\left(h, j_{1}, j_{2}\right)$ lies in the image of $\mathcal{E} \mathcal{M}(23)$ (and thus $\left(h, j_{2}\right)$ lies in the image of $\mathcal{E} \mathcal{M}_{j_{1}}(52)$ ), it follows that $\mathcal{E M}_{j_{1}}^{-1}\left(h, j_{2}\right)$ is connected. At the critical value $\left(2 j_{1}, j_{1}\right)$ of $\mathcal{E M}_{j_{1}}$ we have already observed that, reconstructing the critical $2 j_{1}$-level set of the fully reduced Hamiltonian $H_{j_{1}, j_{1}}$ on $P_{j_{1}, j_{1}}$, we obtain a once pinched 2-torus, which is the critical fiber $\mathcal{E M}_{j_{1}}^{-1}\left(2 j_{1}, j_{1}\right)$. Thus we may apply the monodromy theorem. We find that over a loop $\Gamma$ in the set of regular values in the image of $\mathcal{E} \mathcal{M}_{j_{1}}$, where $\Gamma$ encircles the critical value $\left(2 j_{1}, j_{1}\right)$, the 2-torus bundle $\mathcal{E} \mathcal{M}_{j_{1}}^{-1}(\Gamma)$ has monodromy $\left(\begin{array}{ll}1 & 1 \\ 0 & 1\end{array}\right)$. 


\section{The swing plane angle}

In the original phase space $T^{*} \mathbb{R}^{3}$ with canonical coordinates $\left(x, y, z, p_{x}, p_{y}, p_{z}\right)$ let us project the motion of the swing spring onto the $(x, y)$ plane of its configuration space $\mathbb{R}^{3}$. Taking into account only the relevant quadratic terms

$$
H_{x y}^{0}=\frac{1}{2}\left(p_{x}^{2}+p_{y}^{2}+x^{2}+y^{2}\right)
$$

of the Hamiltonian $\bar{H}$ (17) of the resonant swing spring, we obtain the Hamiltonian of a two degree of freedom harmonic oscillator. The projected motion of this oscillator is an ellipse $E$. For the interpretation of the solutions of the resonant swing spring we need some elementary facts about these ellipses which are the content of the following

Lemma 1. The center of $E$ is at the origin and its major axis makes an angle

$$
\vartheta=\frac{1}{2} \tan ^{-1} \frac{2\left(x y+p_{x} p_{y}\right)}{p_{x}^{2}+x^{2}-p_{y}^{2}-y^{2}}
$$

with the $x$-axis, assuming that $p_{x}^{2}+x^{2}>p_{y}^{2}+y^{2}$. Otherwise, $\vartheta$ is the angle between the major axis and the $y$-axis. In the coordinates $\left(\xi, \eta, \zeta, p_{\xi}, p_{\eta}, p_{\zeta}\right)$ on $\left(T^{*} \mathbb{R}^{3}, \widehat{\omega}\right)$ this angle is

$$
\widetilde{\vartheta}=\Psi^{*} \vartheta=\frac{1}{2} \tan ^{-1} \frac{\xi \eta+p_{\xi} p_{\eta}}{\xi p_{\eta}-\eta p_{\xi}}=\frac{\pi}{2}-\frac{1}{2} \tan ^{-1} \frac{\xi}{p_{\xi}}+\frac{1}{2} \tan ^{-1} \frac{\eta}{p_{\eta}} .
$$

The area of the ellipse $E$ is given by $\pi\left(x p_{y}-y p_{x}\right)^{2}=\pi L^{2}$ and its eccentricity is

$$
\mathrm{e}(E)=2\left(\kappa+\kappa^{-1}\right)^{-1}, \quad \kappa^{4}=\frac{2 j_{1}-\rho_{6}}{2 j_{2}-\rho_{6}} .
$$

Proof. Recall that (see [2])

$$
\begin{array}{ll}
\tau_{1}=x^{2}+p_{x}^{2} & \tau_{3}=x y+p_{x} p_{y} \\
\tau_{2}=y^{2}+p_{y}^{2} & \tau_{4}=x p_{y}-y p_{x} .
\end{array}
$$

are integrals of the 2-dimensional harmonic oscillator vector field

$$
X_{H_{x y}^{0}}=p_{x} \frac{\partial}{\partial x}+p_{y} \frac{\partial}{y}-x \frac{\partial}{\partial p_{x}}-y \frac{\partial}{\partial p_{y}} .
$$

Moreover they satisfy the relation

$$
\tau_{3}^{2}+\tau_{4}^{2}=\tau_{1} \tau_{2}, \quad \tau_{1} \geq 0, \tau_{2} \geq 0
$$


The projection of a motion of the harmonic oscillator with initial condition $\left(x^{0}, y^{0}, p_{x}^{0}, p_{y}^{0}\right)$ and positive energy onto the $(x, y)$-plane is the curve

$$
\gamma: t \mapsto \gamma(t)=(x(t), y(t))=\left(x \cot t+p_{x} \sin t, y \cos t+p_{y} \sin t\right) .
$$

The initial condition determines the values of the integrals $\tau_{i}$, say $\tau_{i}^{0}$. Then

$$
\left(\tau_{1}^{0}-x(t)^{2}\right)\left(\tau_{2}^{0}-y(t)^{2}\right)=\left(p_{x}(t) p_{y}(t)\right)^{2}=\left(\tau_{3}^{0}-x(t) y(t)\right)^{2},
$$

which after some simplification gives

$$
\tau_{2}^{0} x(t)^{2}-2 \tau_{3}^{0} x(t) y(t)+\tau_{1}^{0} y(t)^{2}=\tau_{1}^{0} \tau_{2}^{0}-\left(\tau_{3}^{0}\right)^{2}=\left(\tau_{4}^{0}\right)^{2} .
$$

Since $\tau_{1}^{0}+\tau_{2}^{0}=2 H_{x y}^{0}>0$ and $\tau_{1}^{0} \tau_{2}^{0}-\left(\tau_{3}^{0}\right)^{2} \geq 0$, the quadratic form

$$
(x, y) \mathcal{Q}\left(\begin{array}{l}
x \\
y
\end{array}\right)=(x, y)\left(\begin{array}{rr}
\tau_{2}^{0} & -\tau_{3}^{0} \\
-\tau_{3}^{0} & \tau_{1}^{0}
\end{array}\right)\left(\begin{array}{l}
x \\
y
\end{array}\right)=\left(\tau_{4}^{0}\right)^{2}
$$

is positive definite. Hence the image of the curve $\gamma$ is an ellipse $E$.

Changing coordinates by $\left(\begin{array}{l}x \\ y\end{array}\right)=\left(\begin{array}{cc}\cos \theta & -\sin \theta \\ \sin \theta & \cos \theta\end{array}\right)\left(\begin{array}{l}\xi \\ \eta\end{array}\right)$ transforms the quadratic form $\mathcal{Q}$ (61) into the quadratic form

$$
(\xi, \eta)\left(\begin{array}{cc}
c^{2} \tau_{2}^{0}+s^{2} \tau_{1}^{0}-2 c s \tau_{3}^{0} & c s\left(\tau_{1}^{0}-\tau_{2}^{0}\right)-\left(c^{2}-s^{2}\right) \tau_{3}^{0} \\
c s\left(\tau_{1}^{0}-\tau_{2}^{0}\right)-\left(c^{2}-s^{2}\right) \tau_{3}^{0} & s^{2} \tau_{2}^{0}+c^{2} \tau_{1}^{0}+2 c s \tau_{3}^{0}
\end{array}\right)\left(\begin{array}{l}
\xi \\
\eta
\end{array}\right)
$$

where $c=\cos \theta$ and $s=\sin \theta$. Suppose that $\tau_{1}^{0}>\tau_{2}^{0}$. Choose $\theta$ so that

$$
\theta=\frac{1}{2} \tan ^{-1} \frac{2 \tau_{3}^{0}}{\tau_{1}^{0}-\tau_{2}^{0}}
$$

Then the quadratic form (62) becomes $\lambda_{-} \xi^{2}+\lambda_{+} \eta^{2}=\left(\tau_{4}^{0}\right)^{2}$, where $\lambda_{ \pm}$are eigenvalues of $\mathcal{Q}$, that is,

$$
\lambda_{ \pm}=\frac{1}{2}\left[\left(\tau_{1}^{0}+\tau_{2}^{0}\right) \pm \sqrt{\left(\tau_{1}^{0}+\tau_{2}^{0}\right)^{2}-4\left(\tau_{4}^{0}\right)^{2}}\right]>0 .
$$

Since $\lambda_{+}>\lambda_{-}>0$, a piece of the $\xi$-axis is the major axis of $E$ and $\theta$ (63) is the angle between the major axis and the $x$-axis. Note that the eccentricity $e$ of the ellipse $E$ is $e^{2}=1-\frac{\lambda_{-}}{\lambda_{+}}$. 


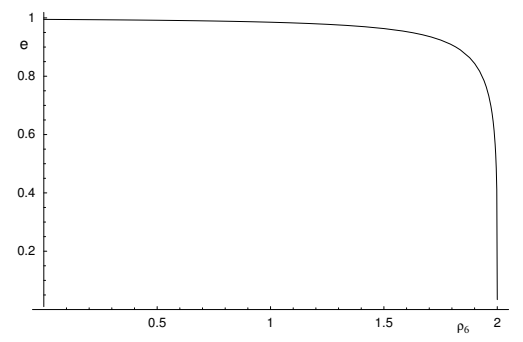

Figure 4: The eccentricity $e\left(\rho_{6}\right)$ for $j_{1}=1, j_{2}=1.5$. The graph is even closer to one when $j_{1}$ and $j_{2}$ are closer to each other.

We now compute $\kappa$. By definition

$$
\begin{aligned}
\kappa^{4} & =\frac{2 j_{1}-\rho_{6}}{2 j_{2}-\rho_{6}}=\frac{\rho_{1}}{\rho_{2}}=\frac{\xi^{2}+p_{\xi}^{2}}{\eta^{2}+p_{\eta}^{2}} \\
& =\frac{x^{2}+p_{x}^{2}+y^{2}+p_{y}^{2}-2\left(x p_{y}-y p_{x}\right)}{x^{2}+p_{x}^{2}+y^{2}+p_{y}^{2}+2\left(x p_{y}-y p_{x}\right)}=\frac{\tau_{1}^{0}+\tau_{2}^{0}-2 \tau_{4}^{0}}{\tau_{1}^{0}+\tau_{2}^{0}+2 \tau_{4}^{0}} \\
& =\frac{\left(\tau_{1}^{0}+\tau_{2}^{0}\right)^{2}-2\left(\tau_{4}^{0}\right)^{2}}{\left(\tau_{1}^{0}+\tau_{2}^{0}+2 \tau_{4}^{0}\right)^{2}} .
\end{aligned}
$$

So

$$
\begin{aligned}
\kappa^{2} & =\frac{\sqrt{\left(\tau_{1}^{0}+\tau_{2}^{0}\right)^{2}-2\left(\tau_{4}^{0}\right)^{2}}}{\tau_{1}^{0}+\tau_{2}^{0}+2 \tau_{4}^{0}} \\
& =\frac{\lambda_{+}-\lambda_{-}}{\lambda_{+}+\lambda_{-}+2 \sqrt{\lambda_{+} \lambda_{-}}}, \quad \begin{array}{l}
\text { since the characteristic polynomial } \\
\text { of } \lambda^{2}-\left(\tau_{1}^{0}+\tau_{2}^{0}\right) \lambda+\left(\tau_{4}^{0}\right)^{2}
\end{array} \\
& =\frac{1-\sqrt{\frac{\lambda_{-}}{\lambda_{+}}}}{1+\sqrt{\frac{\lambda_{-}}{\lambda_{+}}}}
\end{aligned}
$$

Therefore

$$
1-e^{2}=\frac{\lambda_{+}}{\lambda_{-}}=\frac{\left(1-\kappa^{2}\right)^{2}}{\left(1+\kappa^{2}\right)^{2}},
$$

which implies that $e^{2}=\frac{4 \kappa^{2}}{\left(1+\kappa^{2}\right)^{2}}$, that is, $e=\frac{2 \kappa}{1+\kappa^{2}}=\frac{2}{\kappa+\kappa^{-1}}$.

For small but non-zero angular momentum $l$ the motion goes from a small circle to a very eccentric ellipse, see Figure 4 . When $l=0$ then $j_{1}=j_{2}$, hence $\kappa=1$ and $\mathrm{e}=1$ : the motion takes plane on a line, always. However, when 
$j_{1} \neq j_{2}$ then the eccentricity will move between 0 and $1-2 l / j$. $E$ is close to 1 for most values of $\rho_{6}$, and $\vartheta$ gives the angle of the swing plane. Only when $\rho_{6}$ is near its maximum the eccentricity becomes small. For the full Hamiltonian $\bar{H}$ of the swing spring, the angle $\vartheta$ is no longer constant, but instead is slowly varying. In this situation, $\vartheta$ is the instantaneous angle of the swing plane. Similarly $\rho_{6}$ is changing in time, so $e$ becomes the instantaneous eccentricity. Even in the full dynamics the instantaneous ellipse $E$ has constant area $\pi L^{2}$, because $L$ is the conserved angular momentum. In general the eccentricity of $E$ changes during the motion of the swing spring. However, since $\mathrm{e}(E)$ only depends on $\rho_{6}$, we see that it is constant for the critical 2-tori corresponding to a stable equilibrium point of the fully reduced vector field $X_{H_{j_{1}, j_{2}}}$. The swing angle $\vartheta$ should not be confused with the polar angle of polar coordinates in the $(x, y)$-plane, even though $\vartheta$ is canonically conjugate to $L$. As we will see the angle $\vartheta$ commutes with $H_{0}$, which is not true for the polar angle.

We now construct the action-angle variables corresponding to the $T^{2}$ symmetry group. In section 3, we have found a Hamiltonian 2-torus action (24) on $T^{*} \mathbb{R}^{3}$ with momentum map $J=\left(J^{1}, J^{2}\right)(25)$. For a regular value $\left(j_{1}, j_{2}\right)$ of $J$, the two-torus action on the level set $J^{-1}\left(j_{1}, j_{2}\right)=\left(J^{1}\right)^{-1}\left(j_{1}\right) \cap\left(J^{2}\right)^{-1}\left(j_{2}\right)$ is free. Hence we obtain a smooth 2 -torus bundle $\pi_{j_{1}, j_{2}}: J^{-1}\left(j_{1}, j_{2}\right) \rightarrow P_{j_{1}, j_{2}}$ over the fully reduced space $P_{j_{1}, j_{2}}$. We would like to find action-angle coordinates for the 2 -torus fiber $F_{p}=\pi_{j_{1}, j_{2}}^{-1}(p)$ over the point $p \in P_{j_{1}, j_{2}}$. The Hamiltonian vector fields $X_{J^{1}}$ and $X_{J^{2}}$ on $\left(T^{*} \mathbb{R}^{3}, \widehat{\omega}\right)$ leave each 2-torus $F_{p}$ invariant and have only periodic orbits of period $2 \pi$ on $F_{p}$. The same is therefore true for $\widehat{H}_{0}=J^{1}+J^{2}$ and $\widehat{L}=J^{2}-J^{1}$. Since we are interested in the angle $\theta$, we use $\widehat{H}_{0}$ and $\widehat{L}$ as action variables instead of $J^{1}$ and $J^{2}$. We denote the values of these actions by $j$ and $l$, respectively. This leads to the following

Lemma 2. For all regular values of $J$ (25) the variables $\left(I_{1}, \theta_{1}, I_{2}, \theta_{2}\right)$ given by $I_{1}=\widehat{H}_{0}, I_{2}=\widehat{L}$, (see (19) and (20)),

$$
\theta_{1}=\frac{1}{2} \tan ^{-1} \frac{\zeta}{p_{\zeta}}, \quad \theta_{2}=\frac{1}{2} \tan ^{-1} \frac{\eta}{p_{\eta}}-\frac{1}{2} \tan ^{-1} \frac{\xi}{p_{\xi}}
$$

and $\rho_{4}, \rho_{5}, \rho_{6}$ on $P_{j_{1}, j_{2}}$ are a coordinate system for an open subset of phase space $T^{*} \mathbb{R}^{3}$. In these coordinates, the Hamiltonian of the resonant swing spring is

$$
H=I_{1}+\lambda \rho_{4}
$$


Moreover, the following Poisson bracket relations hold.

$$
\begin{aligned}
& \left\{\theta_{1}, I_{1}\right\}=1, \quad\left\{\theta_{2}, I_{2}\right\}=1, \\
& \left\{\theta_{1}, \rho_{4}\right\}=\frac{1}{2} \frac{\rho_{4}}{\rho_{6}}, \quad\left\{\theta_{1}, \rho_{5}\right\}=\frac{1}{2} \frac{\rho_{5}}{\rho_{6}}, \\
& \left\{\theta_{2}, \rho_{4}\right\}=-\frac{\rho_{4} I_{2}}{\rho_{1} \rho_{2}}, \quad\left\{\theta_{2}, \rho_{5}\right\}=-\frac{\rho_{5} I_{2}}{\rho_{1} \rho_{2}}, \\
& \left\{\theta_{1}, \rho_{6}\right\}=1, \quad\left\{\rho_{4}, \rho_{5}\right\}=\rho_{6}\left(\rho_{1}+\rho_{2}\right)-\rho_{1} \rho_{2}, \\
& \left\{\rho_{5}, \rho_{6}\right\}=2 \rho_{4}, \quad\left\{\rho_{4}, \rho_{6}\right\}=-2 \rho_{5},
\end{aligned}
$$

where $\rho_{1}=I_{1}-I_{2}-\rho_{6}$ and $\rho_{2}=I_{1}+I_{2}-\rho_{6}$. All other brackets vanish.

Proof. This last assertion can be verified by direct calculation using the old variables $\left(\xi, \eta, \zeta, p_{\xi}, p_{\eta}, p_{\zeta}\right)$. For example

$$
\begin{aligned}
\left\{\theta_{2}, I_{1}\right\} & =\left(p_{\zeta} \frac{\partial}{\partial \zeta}-\zeta \frac{\partial}{\partial p_{\zeta}}\right) \tan ^{-1} \frac{p_{\zeta}}{\zeta}=1 . \\
\left\{\theta_{1}, I_{2}\right\} & =0 \\
\left\{\theta_{2}, I_{1}\right\} & =\left(+p_{\xi} \frac{\partial}{\partial \xi}+p_{\eta} \frac{\partial}{\partial \eta}-\xi \frac{\partial}{\partial p_{\xi}}-\eta \frac{\partial}{\partial p_{\eta}}\right) \theta_{2}=\frac{1}{2}-\frac{1}{2}=0 \\
\left\{\theta_{2}, I_{2}\right\} & =\left(-p_{\xi} \frac{\partial}{\partial \xi}+p_{\eta} \frac{\partial}{\partial \eta}+\xi \frac{\partial}{\partial p_{\xi}}-\eta \frac{\partial}{\partial p_{\eta}}\right) \theta_{2}=1 \\
\left\{\theta_{1}, \rho_{4}\right\} & =\frac{\left(\xi \eta-p_{\xi} p_{\eta}\right) p_{\zeta}-\left(\xi p_{\eta}+\eta p_{\xi}\right) \zeta}{2\left(p_{\zeta}^{2}+\zeta^{2}\right)}=\frac{\rho_{4}}{2 \rho_{6}} \\
\left\{\theta_{2}, \rho_{4}\right\} & =\frac{\left(p_{\xi}^{2}+\xi^{2}-p_{\eta}^{2}-\eta^{2}\right)\left(\left(\xi \eta-p_{\xi} p_{\eta}\right) p_{\zeta}-\left(\xi p_{\eta}+\eta p_{\xi}\right) \zeta\right)}{2\left(p_{\xi}^{2}+\xi^{2}\right)\left(p_{\eta}^{2}+\eta^{2}\right)}=-\frac{\rho_{4} I_{2}}{\rho_{1} \rho_{2}},
\end{aligned}
$$

and similarly for the other brackets. That all brackets between the new variables can be expressed in terms of the $\rho_{i}$ follows from the fact that

1) $I_{1}$ and $I_{2}$ are actions;

2) the flow of the Hamiltonian vector fields of $I_{1}$ and $I_{2}$ leaves $\rho_{i}$ invariant;

3 ) the actions $I_{1}$ and $I_{2}$ and the angles $\theta_{1}, \theta_{2}$ form a symplectic coordinate system in the two-torus fibre of the bundle $\pi_{j_{1}, j_{2}}$.

Lemma 2 allows us to give a description of the motion of the resonant swing spring in phase space in such a way that the fully reduced system is an invariant subsystem that is driving the dynamics in the fibres. Using the 
fact that $\lambda \rho_{4}=h-j$, the equations of motion for the angles in the fibre are

$$
\begin{aligned}
& \dot{\theta}_{1}=\left\{\theta_{1}, H\right\}=1+\frac{h-j}{2 \rho_{6}}, \\
& \dot{\theta}_{2}=\left\{\theta_{2}, H\right\}=\frac{(h-j) l}{\left(\rho_{6}-j-l\right)\left(\rho_{6}-j+l\right)} .
\end{aligned}
$$

They are driven by the solution of the the second order differential equation

$$
\ddot{\rho}_{6}=2 \lambda^{2} P_{3}^{\prime}\left(\rho_{6}\right)=2 \lambda^{2}\left(2 \rho_{6}\left(j-\rho_{6}\right)-\left(\rho_{6}-j-l\right)\left(\rho_{6}-j+l\right)\right)
$$

Equations (68b) and (69) can be found in [5]. We rederived them because in [5] they were derived under the hypothesis of "pattern evocation in shape space", which might have been an approximation. From our derivation we see that no approximation is involved and that the angle of the swing plane is simply an angle of an action-angle coordinate system.

\section{Actions and Rotation Numbers}

We are interested in the change of $\theta_{2}$ over one period of the motion of $\rho_{6}$. First we directly derive an integral for this change $\Delta \theta_{2}$ by changing the time parametrization in (68b). Then we will show that $\Delta \theta_{2}$ is a rotation number.

The integral curve in the fully reduced system is given by the $h$-level set of the fully reduced Hamiltonian $H_{j_{1}, j_{2}}$ (66) on the fully reduced space $P_{j_{1}, j_{2}}$ (30). Hence the motion takes place on a family of real affine elliptic curves $\mathcal{E}_{h, j_{1}, j_{2}}$ defined by

$$
\rho_{5}^{2}=Q\left(\rho_{6}\right)=-\frac{1}{\lambda^{2}}(h-j)^{2}+\rho_{6}\left(\rho_{6}-j-l\right)\left(\rho_{6}-j+l\right),
$$

when $0 \leq \rho_{6} \leq \min \left(2 j_{1}, 2 j_{2}\right)$. Equation (70) is obtained by eliminating $\rho_{4}$ from (25) using $h=j_{1}+j_{2}+\lambda \rho_{4}$ (which defines $H_{j_{1}, j_{2}}^{-1}(h)$ ). This exactly reproduces the polynomial $Q$ already defined in (36). Therefore also the analysis of the double roots done for the energy momentum map also applies here. The equation (69) for $\rho_{6}$ can be integrated once and we find

$$
\dot{\rho}_{6}=2 \lambda \rho_{5}=2 \lambda \sqrt{Q\left(\rho_{6}\right)} .
$$

Note that this is one of the fully reduced equations of motion (35). When $\left(h, j_{1}, j_{2}\right)$ is a regular value of the energy momentum mapping $\mathcal{E M}$ (23) of 
the swing spring (which we henceforth assume), then the $\mathcal{E}_{h, j_{1}, j_{2}}$ is smooth. When $h=j$ the polynomial $P_{3}\left(\rho_{6}\right)=\rho_{6}\left(j+l-\rho_{6}\right)\left(j-l-\rho_{6}\right)$ is nonnegative on $\left[0, \min \left(2 j_{1}, 2 j_{2}\right)\right]$. Therefore the polynomial $Q$ has three distinct real roots

$$
0 \leq \rho_{6}^{-}<\rho_{6}^{+} \leq \min \left(2 j_{1}, 2 j_{2}\right)<\rho_{6}^{0} .
$$

The motion of the fully reduced system on $H^{-1}(h)$ takes place when $\rho_{6}$ lies in $\left[\rho_{6}^{-}, \rho_{6}^{+}\right]$. Since $H_{j_{1}, j_{2}}^{-1}(h)$ is diffeomorphic to a circle, when the fully reduced motion runs through a period the time parameter $\rho_{6}$ traverses the interval $\left[\rho_{6}^{-}, \rho_{6}^{+}\right]$forward and backward once. Accordingly the period of the driving motion is given by

$$
T=2 \int_{\rho_{6}^{-}}^{\rho_{6}^{+}} \frac{\mathrm{d} \rho_{6}}{2 \lambda \rho_{5}}
$$

To find the change of $\theta_{2}$ over this period, we introduce in (68a) and (68b) a new time scale $\rho_{6}$ defined by (71). We obtain

$$
\begin{aligned}
\mathrm{d} \theta_{1} & =\left(1+\frac{h-j}{2 \rho_{6}}\right) \frac{\mathrm{d} \rho_{6}}{2 \lambda \rho_{5}} \\
\mathrm{~d} \theta_{2} & =\frac{(h-j) l}{\left(j+l-\rho_{6}\right)\left(j-l-\rho_{6}\right)} \frac{\mathrm{d} \rho_{6}}{2 \lambda \rho_{5}}
\end{aligned}
$$

which are differential forms on $\mathcal{E}_{h, j_{1}, j_{2}}$. Therefore the change in the swing angle during a period of the motion of the swing spring is

$$
\begin{aligned}
\Delta \theta_{2}(h, j, l) & =2 \int_{\rho_{6}^{-}}^{\rho_{6}^{+}} \mathrm{d} \theta_{2} \\
& =\frac{(h-j) l}{\lambda} \int_{\rho_{6}^{-}}^{\rho_{6}^{+}} \frac{1}{\left(j+l-\rho_{6}\right)\left(j-l-\rho_{6}\right)} \frac{\mathrm{d} \rho_{6}}{\sqrt{Q\left(\rho_{6}\right)}} .
\end{aligned}
$$

To see that $\Delta \theta_{2}$ is in fact a rotation number of the integrable system $\left(\widehat{H}, J^{1}, J^{2}, T^{*} \mathbb{R}^{3}, \widehat{\omega}\right)$, we have to compute the third action. First we find the angle $\theta_{3}$ conjugate to $\rho_{6}$ on $P_{j_{1}, j_{2}} \backslash\left\{\left(\rho_{6}^{ \pm}, 0\right)\right\}$. A calculation shows that

$$
\theta_{3}=\frac{1}{2} \tan ^{-1} \frac{\rho_{5}}{\rho_{4}}
$$

does the job, because $\left\{\theta_{3}, \rho_{6}\right\}=1$. Therefore, we can define the third action $I_{3}$ as the integral of the canonical one form $\rho_{6} \mathrm{~d} \theta_{3}$ over $\mathcal{E}_{h, j_{1}, j_{2}}$, namely,

$$
I_{3}(h, j, l)=\frac{1}{2 \pi} \oint \rho_{6} \mathrm{~d} \theta_{3}=\frac{h-j}{8 \pi \lambda} \int_{\rho_{6}^{-}}^{\rho_{6}^{+}}\left(3-\frac{2 j_{1}}{\rho_{1}}-\frac{2 j_{2}}{\rho_{2}}\right) \frac{\mathrm{d} \rho_{6}}{\rho_{5}} .
$$


The last equality above is verified as follows. Computing the derivative of $\theta_{3}$ along the integral curves of the fully reduced vector field $X_{j_{1}, j_{2}}$ and using (35) gives

$$
\frac{\mathrm{d} \theta_{3}}{\mathrm{~d} t}=\frac{1}{2} \frac{\rho_{4}}{\rho_{4}^{2}+\rho_{5}^{2}} \frac{\mathrm{d} \rho_{5}}{\mathrm{~d} t}=\frac{h-j}{2 \lambda} \frac{\lambda}{\rho_{1} \rho_{2} \rho_{6}} P_{3}^{\prime}\left(\rho_{6}\right)
$$

So

$$
\frac{\mathrm{d} \theta_{3}}{\mathrm{~d} \rho_{6}}=\frac{h-j}{4 \lambda} \frac{\left(\rho_{1} \rho_{2}-\rho_{2} \rho_{6}-\rho_{1} \rho_{6}\right)}{\rho_{1} \rho_{2} \rho_{5} \rho_{6}} .
$$

Substituting $\rho_{6}=2 j_{1}-\rho_{1}$ and $\rho_{6}=2 j_{2}-\rho_{2}$ gives the equality (76).

The integral (76) implicitly defines the Hamiltonian $H$ as a function of the three actions $I_{1}, I_{2}, I_{3}$. Hence we can obtain the frequencies of the conjugate angles $\theta_{1}, \theta_{2}$, and $\theta_{3}$ by implicit differentiation. In particular we have

$$
\mathrm{d} I_{3}=\frac{\partial I_{3}}{\partial h} \mathrm{~d} H+\frac{\partial I_{3}}{\partial j} \mathrm{~d} I_{1}+\frac{\partial I_{3}}{\partial l} \mathrm{~d} I_{2}
$$

Rewritten this gives

$$
\begin{aligned}
\mathrm{d} H & =-\left(\frac{\partial I_{3}}{\partial h}\right)^{-1} \frac{\partial I_{3}}{\partial j} \mathrm{~d} I_{1}-\left(\frac{\partial I_{3}}{\partial h}\right)^{-1} \frac{\partial I_{3}}{\partial l} \mathrm{~d} I_{2}+\left(\frac{\partial I_{3}}{\partial h}\right)^{-1} \mathrm{~d} I_{3} \\
& =\frac{\partial H}{\partial I_{1}} \mathrm{~d} I_{1}+\frac{\partial H}{\partial I_{2}} \mathrm{~d} I_{2}+\frac{\partial H}{\partial I_{3}} \mathrm{~d} I_{3} .
\end{aligned}
$$

Hence

$$
\dot{\theta}_{3}=\frac{\partial H}{\partial I_{3}}=\left(\frac{\partial I_{3}}{\partial h}\right)^{-1} .
$$

The above expression yields the period $2 \pi \frac{\partial I_{3}}{\partial h}$, which is the same as that given in (72). To see that the integral (72) and the one obtained from (81) give the same result, one has to add a total differential to the integrand of the loop integral (72), which does not contribute to the period. More precisely the identity

$$
\frac{\mathrm{d} \rho_{6}}{\rho_{5}}=\frac{\partial}{\partial h}\left(\frac{h-j}{2}\left(3-\frac{2 j_{1}}{\rho_{1}}-\frac{2 j_{2}}{\rho_{2}}\right) \frac{\mathrm{d} \rho_{6}}{\rho_{5}}\right)+\mathrm{d}\left(\frac{\rho_{6}}{\rho_{5}}\right),
$$

holds on $\mathcal{E}_{h, j_{1}, j_{2}}$, where $\rho_{5}$ is a function of $\rho_{6}$ given by $(70)$. The frequencies of the other angles are similarly obtained as

$$
\dot{\theta}_{2}=\frac{\partial H}{\partial I_{2}}=-\frac{\partial I_{3} / \partial l}{\partial I_{3} / \partial h}
$$




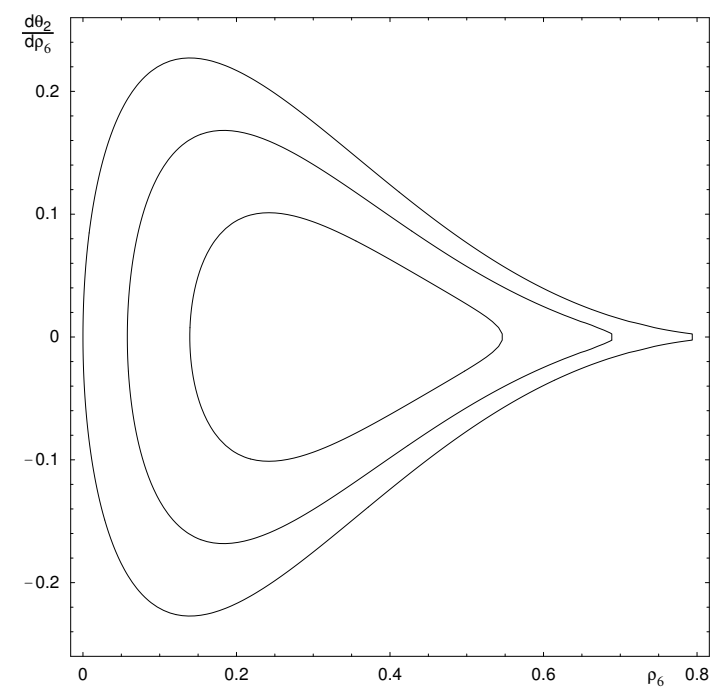

Figure 5: Contour plot of $\chi$ for $j=1, l=0.15$. The contours $0,-0.05,-0.1$ are shown.

and similarly for $\theta_{1}$. Hence we obtain the rotation numbers

$$
W_{23}=\frac{\dot{\theta}_{2}}{\dot{\theta}_{3}}=-\frac{\partial I_{3}}{\partial l}, \text { and } W_{13}=\frac{\dot{\theta}_{1}}{\dot{\theta}_{3}}=-\frac{\partial I_{3}}{\partial j} .
$$

Now we show that $\Delta \theta_{2}=-W_{23}$. The equality holds because the differentials only differ by a total differential, which does not contribute under the closed loop integral. By direct calculation one can check that

$$
\mathrm{d} \theta_{2}=\frac{\partial}{\partial l}\left(\frac{h-j}{4 \lambda}\left(3-\frac{2 j_{1}}{\rho_{1}}-\frac{2 j_{2}}{\rho_{2}}\right) \frac{\mathrm{d} \rho_{6}}{\rho_{5}}\right)+\mathrm{d}\left(\rho_{6} \frac{\mathrm{d} \theta_{2}}{\mathrm{~d} \rho_{6}}\right),
$$

where $\mathrm{d} \theta_{2}$ is given by $(73 \mathrm{~b})$. The first derivation of an integral for the stepwise precession of the swing plane is simpler than the present one. But the latter derivation shows that the stepwise precession of the swing plane is one of the two rotation numbers of the invariant tori of the integrable approximation.

In order to understand the solution curves of the differential equation for $\mathrm{d} \theta_{2} / \mathrm{d} \rho_{6}$ (73b) we can plot its solution curves. This is easily done by finding a function of $\theta_{2}$ and $\rho_{6}$ that is constant on the solutions. Such a function can be defined as

$$
\chi(x, y)=\frac{y^{2}}{\left((x-j)^{2}-l^{2}\right)^{2}}-x\left((x-j)^{2}-l^{2}\right)
$$


where $\chi\left(\rho_{6}, \mathrm{~d} \theta_{2} / \mathrm{d} \rho_{6}\right)=-(h-j)^{2} / \lambda^{2}$. A contour plot of $\chi$ is shown in Figure 5. On the right part of figure the $\rho_{6}$ is large, so that the eccentricity is small. On the left side $\rho_{6}$ is small, hence the eccentricity is close to 1 , see Figure 4 .

\section{Analysis of the swing angle}

In this section we examine more closely our formula (74) for the swing angle of the swing spring. We prove the following

Proposition. The rotation number describing the angle of stepwise precession for the integrable approximation of the resonant swing spring is given by

$$
\Delta \theta_{2}=\tan ^{-1} \frac{l \sqrt{j}}{(h-j) / \lambda}+O\left(\sqrt{l^{2}+((h-j) / \lambda)^{2} / j} / j\right) .
$$

Equation (87) shows that the swing angle $\Delta \theta_{2}$ is a multivalued function of the parameters $h, j_{1}$ and $j_{2}$. From this one can read off that the Liouville integrable system $\left(\widehat{H}, \widehat{L}, \widehat{H}_{0}, T^{*} \mathbb{R}^{3}, \widehat{\omega}\right)$ describing the swing spring has monodromy.

Proof. In order to simplify our analytical study of (74) we remove an unneeded parameter by the rescaling

$$
\rho_{6}=j \tilde{z}, \quad l=j \widetilde{b} \quad \text { and } \quad \widetilde{a} j^{3 / 2}=\frac{h-j}{\lambda} .
$$

Equation (74) then becomes

$$
\Delta \theta_{2}=\widetilde{a} \tilde{b} \int_{\tilde{z}^{-}}^{\tilde{z}^{+}} \frac{1}{(1-\tilde{z})^{2}-\widetilde{b}^{2}} \frac{\mathrm{d} \tilde{z}}{\sqrt{\tilde{z}\left((1-\tilde{z})^{2}-\widetilde{b}^{2}\right)-\widetilde{a}^{2}}}
$$

Here $0 \leq \tilde{z}^{-}<\tilde{z}^{+} \leq 1<\tilde{z}^{0}$ are distinct nonnegative roots of the polynomial

$$
\tilde{z}\left(\left(1-\tilde{z}^{2}\right)-\widetilde{b}^{2}\right)-\widetilde{a}^{2} .
$$

Next we restrict the parameters $\widetilde{a}$ and $\widetilde{b}$ in (89) to lie on a line through the origin in parameter space $\mathbb{R}^{2}$ and assume that they are both small. In other words, we introduce a small parameter $\varepsilon$ such that

$$
\widetilde{a}=\varepsilon a \quad \text { and } \quad \widetilde{b}=\varepsilon b,
$$


where $(a, b)$ are fixed in parameter space.

Now we find the Taylor expansion of the roots $\widetilde{z}^{-}, \widetilde{z}^{+}$, and $\widetilde{z}^{0}$. A calculation gives

$$
\begin{aligned}
& \widetilde{z}^{-}=a^{2} \varepsilon^{2}+\left(2 a^{4}+a^{2} b^{2}\right) \varepsilon^{4}+\mathrm{O}\left(\varepsilon^{6}\right) \\
& \widetilde{z}^{+}=1-\sqrt{a^{2}+b^{2}} \varepsilon-\frac{1}{2} a^{2} \varepsilon^{2}+\mathrm{O}\left(\varepsilon^{3}\right) \\
& \widetilde{z}^{0}=1+\sqrt{a^{2}+b^{2}} \varepsilon+\frac{1}{2} a^{2} \varepsilon^{2}+\mathrm{O}\left(\varepsilon^{3}\right) .
\end{aligned}
$$

Because the root $\widetilde{z}^{+}$and the pole at $1-\varepsilon b$ coalesc at 1 as $\varepsilon \rightarrow 0$, we introduce a shifted, scaled, and inverted new variable $z$ by

$$
\widetilde{z}=1+\varepsilon b / z .
$$

The inversion ensures that the new integration boundaries are finite in the limit $\varepsilon \rightarrow 0$. Then (89) becomes

$$
\Delta \theta_{2}=\int_{z^{-}}^{z^{+}} \frac{a z^{2}}{z^{2}-1} \frac{\mathrm{d} z}{\sqrt{-z\left[\left(a^{2}+b^{2}\right) z^{3}-b^{2} z+\varepsilon b^{3}\left(z^{2}-1\right)\right]}},
$$

Factor the polynomial under the square root as

$$
-z\left[\left(a^{2}+b^{2}\right) z^{3}-b^{2} z+\varepsilon b^{3}\left(z^{2}-1\right)\right]=\left(z-z^{-}\right)\left(z^{+}-z\right) z\left(z-z^{0}\right)\left(a^{2}+b^{2}\right)
$$

and introduce a new integration variable $\phi$ by

$$
2 z=\left(z^{+}+z^{-}\right)+\left(z^{+}-z^{-}\right) \cos \phi .
$$

The integral (93) becomes

$$
\Delta \theta_{2}=\frac{1}{\sqrt{a^{2}+b^{2}}} \int_{0}^{\pi} f(z(\phi)) \mathrm{d} \phi,
$$

where

$$
f(z)=\frac{a z^{2}}{\left(z^{2}-1\right) \sqrt{z\left(z-z^{0}\right)}} .
$$

Using (92) we find that (91) becomes

$$
\begin{aligned}
& z^{-}=-\frac{b}{\sqrt{a^{2}+b^{2}}} \varepsilon-\frac{a^{2} b}{a^{2}+b^{2}} \varepsilon+\mathrm{O}\left(\varepsilon^{2}\right) \\
& z^{+}=-b \varepsilon+\mathrm{O}\left(\varepsilon^{3}\right) \\
& z^{0}=+\frac{b}{\sqrt{a^{2}+b^{2}}} \varepsilon-\frac{a^{2} b}{a^{2}+b^{2}} \varepsilon+\mathrm{O}\left(\varepsilon^{2}\right) .
\end{aligned}
$$


Expanding the integrand of (95) up through terms of order $\varepsilon$ gives

$$
\frac{-2 a b(\cos \phi-1)^{2}}{\left(4 a^{2}+(3-\cos \phi)(1+\cos \phi) b^{2}\right) \sqrt{(\cos \phi-1)(\cos \phi-3)}}+\mathrm{O}(\varepsilon) \text {. }
$$

Note that the error term is uniformly bounded in the interval of integration. The main purpose of the above transformations was to achieve this. Now the zero order contribution in (95) can be calculated. The substitution $\cos \phi=$ $1+2 \sin \psi$ removes the root and the second substitution $\cos \psi=x$ rationalizes (98). Hence the integral becomes

$$
\int_{0}^{1} \frac{a b \mathrm{~d} x}{a^{2}+b^{2} x^{2}}=\tan ^{-1} \frac{b}{a}
$$

Undoing the scaling gives the desired result.

Using the above method, one can actually compute one more order. But it turns out to be zero. At $O\left(\varepsilon^{2}\right)$ the integrand is not uniformly bounded. So more sophisticated methods are needed to obtain the first nonzero correction. Note that the error term is small when we are close to the thread in the bifurcation diagram. Then $l$ and $h-j$ are close to zero. Of course $j$ itself is also small, since we must be close to the equilibrium, but $l$ and $h-j$ are considered to be much smaller.

The result means that for each fixed value of $j>0$, the swing angle $\Delta \theta_{2}$ is a multivalued function of $l$ and $h$. This is another proof of the fact that the system has monodromy. The resonant swing spring provides an example in which the monodromy can be easily observed.

\section{Acknowledgements}

This work was supported by the EU network HPRN-CT-2000-0113 MASIE Mechanics and Symmetry in Europe. A first draft of this paper was written at University of Warwick during the Symposium on Geometric Mechanics and Symmetry 2002. The authors would like to thank the Maths Research Centre at Warwick for its hospitality. 


\section{References}

[1] V. I. Arnold (Ed.), Dynamical Systems III, Springer, Heidelberg, 1988.

[2] R. Cushman and L. Bates, Global Aspects of Classical Integrable Systems, Birkhäuser, Basel, 1997.

[3] R. Cushman and J.J. Duistermaat, Non-Hamiltonian monodromy, J. Diff. Eqns. 172 (2001) 42-58.

[4] J.J. Duistermaat, On global action angle coordinates, Comm. Pure Appl. Math. 33 (1980) 687-706.

[5] D.D. Holm and P. Lynch, Stepwise precession of the resonant swinging spring, SIAM J. Appl. Dynamical Systems 1 (2002) 44-64.

[6] P. Lynch, The swinging spring: a simple model for atmospheric balance. Proceedings of the Symposium on the Mathematics of AtmosphereOcean Dynamics Isaac Newton Institute, June-December, 1996. Cambridge University Press.

[7] P. Lynch, Resonant motions of the three-dimensional elastic pendulum. Intl. J. Nonlin. Mech 37 (2001) 345-367

[8] J. E. Marsden and J Scheurle, Pattern evocation and geometric phases in mechanical systems with symmetry. Dynam. Stability Stystems 10(4) (1995) 315-338

[9] V. Matveev, Integrable Hamiltonian systems with two degrees of freedom. The topological structure of saturated neighborhoods of points of focus-focus and saddle-saddle type. Sb. Math. 187 (1996) 495-524.

[10] San Vu Ngoc, On semi-global invariants for focus-focus singularities, preprint Grenoble 2001.

[11] A. Vitt and G. Gorelik, Oscillations of an elastic pendulum as an example of the oscillations of two parametrically coupled linear systems (in Russian), Zh. Tekh. Fiz. 3(2-3) (1933) 294-307 = English translation by Lisa Shields, with an introduction by Peter Lynch. Historical Note No. 3, Met Eireann, Dublin (1999).

[12] N.T. Zung, A note on focus-focus singularities, Diff. Geom. Appl. 7 (1997) 123-130. 